\title{
The Performance of Wireless Powered MIMO Relaying with Energy Beamforming
}

DOI:

10.1109/TCOMM.2016.2606619

\section{Document Version}

Accepted author manuscript

Link to publication record in Manchester Research Explorer

\section{Citation for published version (APA):}

Almradi, A., \& Hamdi, K. (2016). The Performance of Wireless Powered MIMO Relaying with Energy

Beamforming. IEEE Transactions on Communications, 64(11), 4550-4562.

https://doi.org/10.1109/TCOMM.2016.2606619

\section{Published in:}

IEEE Transactions on Communications

\section{Citing this paper}

Please note that where the full-text provided on Manchester Research Explorer is the Author Accepted Manuscript or Proof version this may differ from the final Published version. If citing, it is advised that you check and use the publisher's definitive version.

\section{General rights}

Copyright and moral rights for the publications made accessible in the Research Explorer are retained by the authors and/or other copyright owners and it is a condition of accessing publications that users recognise and abide by the legal requirements associated with these rights.

\section{Takedown policy}

If you believe that this document breaches copyright please refer to the University of Manchester's Takedown Procedures [http://man.ac.uk/04Y6Bo] or contact uml.scholarlycommunications@manchester.ac.uk providing relevant details, so we can investigate your claim.

\section{OPEN ACCESS}




\title{
The Performance of Wireless Powered MIMO Relaying with Energy Beamforming
}

\author{
Ahmed Almradi and Khairi Ashour Hamdi
}

\begin{abstract}
This paper analyzes the performance of energyconstrained dual-hop amplify-and-forward (AF) relaying systems with multi-antenna nodes, in the presence of multiple co-channel interferers (CCI) at the destination. To maximize the overall signal-to-interference-plus-noise ratio (SINR) as well as the harvested energy so as to mitigate the severe effects of fading and enable long-distance wireless power transfer, hop-by-hop information and energy beamforming is proposed where the transmitted signal is steered along the strongest eigenmode of each hop. The wirelessly powered relay scavenge energy from the source information radio-frequency (RF) signal through energy beamforming, where both the time-switching receiver (TSR) and power-splitting receiver (PSR) are considered, then uses the harvested energy to forward the source message to the destination. To this end, tight lower and upper bound expressions for the outage probability and ergodic capacity are presented in closed-form. These are employed to investigate the throughput of the delay-constrained and delay-tolerant transmission modes. In addition, the asymptotic high signal-to-noise ratio (SNR) outage probability and ergodic capacity approximations are derived, where the achievable diversity order is also presented. Numerical results sustained by Monte Carlo simulations show the tightness of the proposed analytical expressions. The impact of various parameters such as energy harvesting time, power-splitting ratio, source transmit power and the number of antennas on the system throughput is also considered.
\end{abstract}

Index Terms-MIMO relaying, half-duplex relaying, beamforming, maximum ratio transmission (MRT), maximum ratio combining (MRC), zero-forcing (ZF), wireless power transfer, energy harvesting, outage probability, ergodic capacity.

\section{INTRODUCTION}

C ONVENTIONAL wireless information transmission systems have a limited viable lifetime and require periodical battery recharging or replacement, which besides being costly, it can be undesirable or sometimes not feasible. Energy harvesting techniques, which rely on external natural resources such as solar and wind energy, have attracted a lot of research attention due to its ability to prolong the life time of wireless communication systems. However, the naturally harvested energy is random and highly dependent on the weather conditions, thus, impinging on the whole reliability of wireless communications. Recently, wireless power transfer (WPT) is introduced to overcome such problem, where energy

A. Almradi is with the Department of Electrical and Electronic Engineering, Azzaytuna University, Tarhuna, Libya. He is also with the school of Electrical and Electronic Engineering, The University of Manchester, Manchester, UK. (e-mail: elmaradi@gmail.com).

K. A. Hamdi is with the school of Electrical and Electronic Engineering, The University of Manchester, Manchester, UK. (e-mail: k.hamdi@manchester.ac.uk).

Part of this paper was presented at the IEEE International Conference on Communications (ICC), Kuala Lumpur, Malaysia, May 2016. is harvested through ambient radio-frequency (RF) signals [1][6].

Due to practical circuit limitations, the receiver can not decode information and harvest energy from the same signal. Therefore, different wireless information and power transfer (WIPT) architectures have been proposed in the literature (see e.g., [1], [2], [7], [8]), namely, time-switching receiver where the receiver switches over time between information decoding and energy harvesting, power-splitting receiver where the receiver splits the signal into two portions, one for information decoding and the other for harvesting energy, and antennaselection receiver in the case of multiple transmit antennas where a subset of the available antennas is used for information decoding while the remaining set is used for energy harvesting. The optimal switching, splitting, or selection ratio which maximizes the overall system throughput is selected.

More recently, wireless information transfer and energy harvesting in the context of cooperative relaying systems received a great deal of attention, where an intermediate relay with limited battery reserves which relies on external charging mechanism in order to assist in delivering the source signal to the destination. The work in [4] investigated the throughput performance of delay-limited and delay-tolerant transmission modes, for an amplify-and-forward (AF) relaying systems, where both time-switching and power-splitting receivers are considered. The work in [4] was extended in [9] by assuming nakagami-m fading channels, and including co-channel interference at the relay and destination nodes. In [3], a decodeand-forward (DF) relaying system with co-channel interference at the relay is considered, where the energy constrained relay harvests energy from both, the received information signal and the co-channel interference signals, whereby the throughput performance of time-switching and power-splitting receivers are studied. The authors in [10] addressed several power allocation strategies where multiple source-destination pairs communicate with each other with the help of energy harvesting relay. The performance analysis of relay selection in a wireless powered cooperative relays has been studied in [11], [12]. Meanwhile, in the context of full-duplex (FD) relaying, the authors in [13] proposed exploiting both antennas at the FD relay (i.e., the receive and transmit antennas) to increase the harvested energy during the power transfer period using the time switching receiver, thus resulting in an improved overall system throughput. However, all these works are limited to single antenna nodes and therefore face numerous challenges. For instance, owing to the fact that wireless power transfer is highly sensitive to channel fading and path loss, wireless information and power transfer systems are more susceptible to multipath channel fading and path loss when compared to the 
conventional self-powered wireless information transmission. Hence, resulting in an extremely short power transfer distance.

The benefits of transmit and receive diversity in wireless information and power transfer are twofold, in addition to combating the severe effects of multipath fading as in the traditional wireless information transmission, it improves the scavenged energy as it also benefits from spatial energy beamforming [1], i.e., steering the transmitted information and/or energy signal along the strongest eigenmodes of the multipleinput multiple-output (MIMO) source $\rightarrow$ relay and relay $\rightarrow$ destination channels in order to maximize the overall signalto-interefernce-plus-noise ratio (SINR). In the conventional point to point communication, the performance limit of MIMO systems with separated and co-located information processing and energy harvesting receivers are investigated in [14]. The authors in [15] and [16] studied the performance analysis of a wireless powered single antenna user communicating with a multi-antenna hybrid access point, where energy is scavenged from RF signal through energy beamforming. In [17], the performance analysis of a wireless powered multi-antenna relay is considered, where different linear processing schemes are presented for the power-splitting relay receiver. Closedform lower and upper bounds are derived for the outage probability and ergodic capacity, respectively. On the other hand, in the context of FD relaying, to improve the overall system throughput, the authors in [18] proposed that the relay is operated in the HD mode during the first half of the block time, and in the FD mode during the second half of the block time, where the loopback self-interference is used as a source of additional energy besides the source signal. However, in all previous relaying system set-ups, one or more terminals are limited to single antenna structure. To the best of the authors knowledge, the performance analysis of hop-by-hop information and energy beamforming, where the source and relay perform eigenmode beamforming, in MIMO cooperative relaying systems has not been investigated yet.

In this paper, motivated by the above mentioned limitations, we investigate the performance analysis of MIMO relaying systems with an energy-constrained AF half-duplex (HD) relay in the presence of multiple interferers at the destination, where hop-by-hop energy and information beamforming are utilized to maximize the harvested energy as well as the overall received SINR, where both the time-switching receiver (TSR) and power-splitting receiver (PSR) are considered. In this paper, we examine the general situation where each node can have an arbitrarily multiple antennas, including multiple cochannel interferers (CCI) at the destination, where we jointly design the precoding and decoding at the source, relay, and destination in order to maximize the overall SINR.

The main contributions of this paper are summarized as follows:

1) The optimization problem for the design of source, relay, and destination precoding and/or decoding weight vectors which maximizes the overall signal-to-interferenceplus-noise ratio (SINR) is formulated, then a sub-optimal solution based on the added receive zero-forcing (ZF) constraint (null space projection designed to completely suppress the CCI at the destination) is proposed for the second-hop, through which a transmit and receive beamforming weight vectors are derived and used to present a closed-form overall SINR.

2) As the performance analysis of the derived exact overall SINR is a challenging mathematical problem due to the presence of high correlation between the first-hop and second-hop SNRs, and the complicated statistics of the second-hop SNR, and as far as a closed-form outage probability and ergodic capacity expressions are concern, a simpler mathematically tractable overall SINR upper-bound is proposed.

3) A new closed-form expression for the outage probability of the proposed overall SINR upper-bound is presented, where it is shown to be a tight lower-bound to the outage probability for the exact overall SINR. In addition, an asymptotically exact outage probability lower-bound expression is derived, by which the characterization of high SNR outage probability show that the achievable diversity order is $\min \left(N_{S} N_{R}, N_{R}\left(N_{D}-M\right)\right)$.

4) A new closed-form approximate ergodic capacity expression for the proposed overall SINR upper-bound is derived. Besides, a tight closed-form lower-bound ergodic capacity expression for the proposed overall SINR upper-bound is also presented. These expressions are shown to be tight over the entire SNR range of interest, and become exact as the SNR and/or number of antennas approaches infinity (i.e., the asymptotic results of these expressions are exact).

The structure of the rest of the paper is as follows. In section II, we introduce the system and channel model. In section III, the instantaneous overall SINR is addressed. In section IV, the performance analysis is considered. Numerical results are provided in section V. Finally, section VI concludes the paper.

\section{The System And Channel Model}

We consider a dual-hop amplify-and-forward wirelessly powered relay system with multi-antenna terminals, where a source $S$ with $N_{S}$ transmitting antennas is communicating with a destination $\boldsymbol{D}$ with $N_{D}$ receiving antennas through an energy-constrained relay $\boldsymbol{R}$ with $N_{R}$ antennas, in the presence of $M$ co-channel interferers (CCI) at the destination ${ }^{1}$ as depicted in Fig. 1(a). Throughout this paper, the following assumptions are considered: 1) It is assumed that the source does not have a direct link to the destination due to heavy path loss and shadowing (i.e., the direct link is assumed to be in deep fade). 2) A single half-duplex AF relay is considered, where communications take place in two orthogonal channels as the relay can not receive and re-transmit information at the same time over the same frequency due to the inherent half-duplex relaying transmission constraint. 3) Channels are modeled as quasi-static block flat fading and remain constant over the block time $T$, and varies independently and identically from one block to the next. The source $\rightarrow$ relay $(S \rightarrow R)$

\footnotetext{
${ }^{1}$ As far as a closed-form performance analysis expression is concern, the problem of incorporating $\mathrm{CCI}$ at the relay is ignored in this paper due to the challenging mathematical tractability of the resultant overall SINR. Note that our analysis could be seen as a bound to the case where CCI is considered at the relay.
} 
channel $\boldsymbol{H}_{1}$ with $N_{R} \times N_{S}$ matrix, the relay $\rightarrow$ destination $(\boldsymbol{R} \rightarrow \boldsymbol{D})$ channel $\boldsymbol{H}_{2}$ with $N_{D} \times N_{R}$ matrix, and the $i^{\text {th }}$ interferer channel at the destination $\boldsymbol{g}_{i}$ with $N_{D} \times 1$ vector represent small scale fading with entries follow independent and identically distributed $(i . i . d$.) random variables distributed according to ${ }^{2} \mathcal{C N}(0,1)$. Full channel state information (CSI) of the $\boldsymbol{S} \rightarrow \boldsymbol{R}$ channel $\boldsymbol{H}_{1}$ is assumed to be available at the source and the relay, while full CSI of the $R \rightarrow D$ channel $\boldsymbol{H}_{2}$ and the $i^{\text {th }}$ interferer channel $\boldsymbol{g}_{i}$ with $i=1, \ldots, M$ are assumed to be available at the relay and destination. 4) The relay is an energy-constrained and relies on energy harvested from the source energy signal in order to assist communication between the source and destination nodes.

In this paper, the performance of both TSR and PSR are investigated, assuming that the relay has no external power supply and all harvested energy from the received signal at every block time $T$ is consumed by the relay to forward the message signal to the destination.

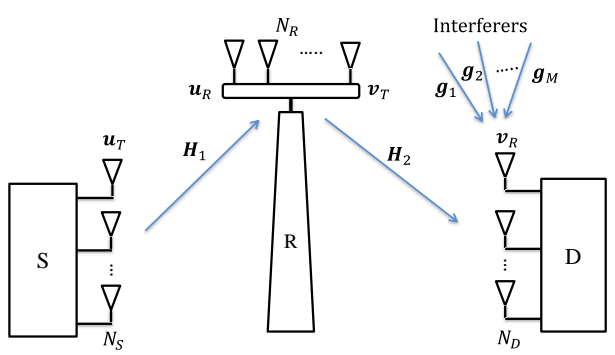

(a)

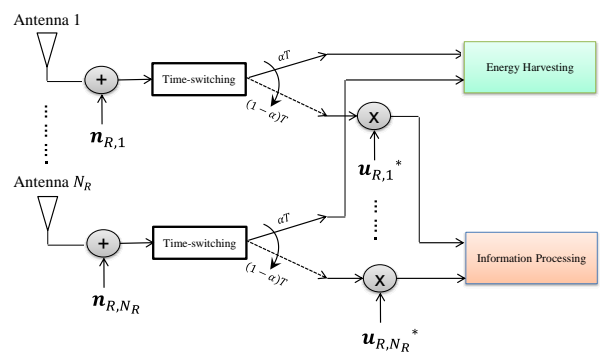

(b)

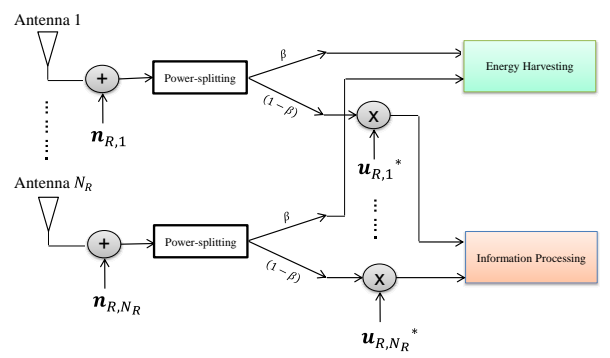

(c)

Figure 1: (a) The MIMO relaying system model. (b) Timeswitching receiver. (c) Power-splitting receiver.

\footnotetext{
${ }^{2}$ It is to be emphasized that Rician fading is used to model the fading when there is a line-of-sight between the transmitter and receiver. However, this paper addresses the challenging problem of long-distance wireless power transfer, where MIMO hop-by-hop information and energy beamforming is exploited to extend the network coverage. Therefore, Rayleigh fading is assumed in this paper.
}

\section{The InSTANTANEOUS Overall SINR}

In this section, the overall SINR for the time-switching receiver and power-splitting receiver are presented. Therefore, as far as a closed-form simple analytically tractable overall SINR is concern, the optimal transmit and receive precoding weight vectors are obtained through solving an optimization problem subject to an added zero forcing constraint that forces the co-channel interferers to zero.

\section{A. Time-Switching Receiver (TSR)}

The time-switching receiver for wireless information and power transfer (WIPT) is depicted in Fig. 1(b). At every block time $T$, where a certain block of information is transmitted from the source node to the destination node, the relay uses a fraction $\alpha$, with $0<\alpha<1$ of the block time to harvest energy from the source energy signal, where the harvested energy is used to forward the source signal to the destination. The remaining block time $(1-\alpha) T$ is divided into two equal parts, one for transmitting the information signal from the $\boldsymbol{S} \rightarrow \boldsymbol{R}$, and the other for transmitting the information signal from the $\boldsymbol{R} \rightarrow \boldsymbol{D}$. All energy harvested during the power transfer phase is consumed by the relay when forwarding the source signal to the destination. The optimal choice for the time fraction $\alpha$ which maximizes the achievable throughput is selected.

The received signal during the energy harvesting phase is given as

$$
\boldsymbol{y}_{E}=\sqrt{\frac{P_{S}}{d_{1}^{\tau}}} \boldsymbol{H}_{1} \boldsymbol{u}_{T} x_{E}+\boldsymbol{n}_{R}
$$

where $P_{S}$ denotes the source power, $d_{1}$ is the distance between the source and relay, with $\tau$ being the path loss exponent, $x_{E}$ is the energy symbol with unit power, $\boldsymbol{u}_{T}$ is the transmit information and energy beamforming weight vector at the source with unit norm, and $\boldsymbol{n}_{R}$ is the noise vector at the relay which is modeled by complex additive white Gaussian noise (AWGN) with zero mean and co-variance of $\sigma^{2} \boldsymbol{I}_{N_{R}}$.

Due to the energy conservation law, the harvested energy is proportional to the energy of the base-band signals, and is directly applied to the received RF signal from all receive antennas. Therefore, once ignoring the negligible energy harvested from the AWGN noise, it is easily shown that the relay transmit power after $\alpha T$ harvesting time can be written as $P_{R}=\eta \frac{\alpha T}{\frac{(1-\alpha) T}{2}} \frac{P_{S}}{d_{1}^{\tau}}\left\|\boldsymbol{H}_{1} \boldsymbol{u}_{T}\right\|^{2}=\eta \frac{2 \alpha}{1-\alpha} \frac{P_{S}}{d_{1}^{\tau}}\left\|\boldsymbol{H}_{1} \boldsymbol{u}_{T}\right\|^{2}$, where $\eta$ is the energy conversion efficiency, and $\|\cdot\|$ denotes the Euclidean norm.

During the first-hop information transmission phase, the combined received signal at the relay is given by

$$
y_{R}=\sqrt{\frac{P_{S}}{d_{1}^{\tau}}} \boldsymbol{u}_{R}^{\dagger} \boldsymbol{H}_{1} \boldsymbol{u}_{T} x_{S}+\boldsymbol{u}_{R}^{\dagger} \boldsymbol{n}_{R}
$$

where $\boldsymbol{u}_{R}$ is the receive combining weight vector at the relay with unit norm, $\dagger$ denotes the conjugate transpose operator, and $x_{S}$ is the information signal with unit power. 
The combined received signal at the destination can be written as

$$
\begin{aligned}
y_{D} & =\sqrt{\frac{P_{R}}{d_{2}^{\tau}}} \mathcal{G} \boldsymbol{v}_{R}^{\dagger} \boldsymbol{H}_{2} \boldsymbol{v}_{T} y_{R}+\sum_{i=1}^{M} \sqrt{\frac{\varrho_{i}}{d_{I i}^{\tau}}} \boldsymbol{v}_{R}^{\dagger} \boldsymbol{g}_{i} y_{i}+\boldsymbol{v}_{R}^{\dagger} \boldsymbol{n}_{D} \\
& =\sqrt{\frac{P_{R}}{d_{2}^{\tau}}} \mathcal{G} \boldsymbol{v}_{R}^{\dagger} \boldsymbol{H}_{2} \boldsymbol{v}_{T} y_{R}+\boldsymbol{v}_{R}^{\dagger} \boldsymbol{G} P_{I}^{\frac{1}{2}} \boldsymbol{y}+\boldsymbol{v}_{R}^{\dagger} \boldsymbol{n}_{D}
\end{aligned}
$$

where $\boldsymbol{v}_{T}$ is the transmit beamforming weight vector at the relay with unit norm, $\boldsymbol{v}_{R}$ is the receive combining weight vector at the destination with unit norm, $d_{2}$ denotes the distance between the relay and the destination, $\varrho_{i}$ denotes the $i^{\text {th }}$ interferer power, $d_{I i}$ denotes the distance between the $i^{\text {th }}$ interferer and the destination, $y_{i}$ is the $i^{\text {th }}$ interferer signal with unit power, $M$ is the number of interferers, $\boldsymbol{n}_{D}$ is the noise at the destination which is modeled by complex AWGN with zero mean and co-variance of $\sigma^{2} \boldsymbol{I}_{N_{D}}$, and $\mathcal{G}$ is the channel assisted (variable gain) relay normalizing constant. Let $\boldsymbol{G}=$ $\left[\boldsymbol{g}_{1}, \boldsymbol{g}_{2}, \ldots, \boldsymbol{g}_{M}\right]$ be the co-channel interference (CCI) channel matrix with $N_{D} \times M$ matrix. Therefore, $\sum_{i=1}^{M} \sqrt{\frac{\varrho_{i}}{d_{I i}^{\tau}}} \boldsymbol{v}_{R}^{\dagger} \boldsymbol{g}_{i} y_{i}$ can be re-written as $\boldsymbol{v}_{R}^{\dagger} \boldsymbol{G} P_{I}^{\frac{1}{2}} \boldsymbol{y}$, where $\boldsymbol{y}$ is the transmitted signal vector of the interferers given by $\boldsymbol{y}=\left[y_{1}, y_{2}, \ldots, y_{M}\right]^{T}$, with $(\cdot)^{T}$ denotes the transpose operator and $P_{I}$ is a diagonal matrix given as $P_{I}=\operatorname{diag}\left(\frac{\varrho_{1}}{d_{I 1}^{\tau}}, \frac{\varrho_{2}}{d_{I 2}^{\tau}}, \ldots, \frac{\varrho_{M}}{d_{I M}^{\tau}}\right)$.

The relay gain for the time-switching receiver is given by

$$
\begin{aligned}
\mathcal{G}^{-1} & =\sqrt{\mathbb{E}\left(\left|y_{R}\right|^{2}\right)} \\
& =\sqrt{\frac{P_{S}}{d_{1}^{\tau}}\left|\boldsymbol{u}_{R}^{\dagger} \boldsymbol{H}_{1} \boldsymbol{u}_{T}\right|^{2}+\sigma^{2}}
\end{aligned}
$$

where $\mathbb{E}(\cdot)$ is the expectation operator, and $|\cdot|$ is the absolute value operator.

From (3) and (4), the overall SINR can be simplified as in (5), shown at the top of next page, where $\rho=\frac{P_{S}}{\sigma^{2}}$.

In order to mitigate the severe effects of fading and enable long-distance wireless power transfer, our objective is to find an optimal transmit and receive precoding weight vectors so that the overall SINR in (5) is maximized. Therefore, the optimization problem may be formulated as

$$
\begin{array}{cc}
\boldsymbol{w}^{\star}=\underset{\substack{\boldsymbol{u}_{T}, \boldsymbol{u}_{R}, \boldsymbol{v}_{T}, \boldsymbol{v}_{R} \\
\text { s. t. }}}{\arg \max } & \gamma(\text { in Eq. (5)) } \\
& \left\|\boldsymbol{u}_{T}\right\|=\left\|\boldsymbol{u}_{R}\right\|=\left\|\boldsymbol{v}_{T}\right\|=\left\|\boldsymbol{v}_{R}\right\|=1
\end{array}
$$

where $\boldsymbol{w}^{\star} \in\left\{\boldsymbol{u}_{T}^{\star}, \boldsymbol{u}_{R}^{\star}, \boldsymbol{v}_{T}^{\star}, \boldsymbol{v}_{R}^{\star}\right\}$. It is well known that in the absence of CCI interference, maximum-ratio transmission (MRT) information and energy beamforming at the transmitter and maximum-ratio combining (MRC) at the receiver are optimal precoding schemes as they result in the maximum overall SINR [19]. However, in the presence of CCI interference, MRT and MRC are sub-optimal as they treat the interference as additive noise. Meanwhile, as far as a closed-form simple analytically tractable overall SINR expression is concern, a sub-optimal solution for the receive combining weight vector at the destination is found by applying the zero forcing (ZF) precoding scheme, where the interference term $P_{I}^{\frac{1}{2}} \boldsymbol{G}^{\dagger} \boldsymbol{v}_{R}$ in (5) is forced to zero assuming that $N_{D}>M$, i.e., we add the ZF constraint $\boldsymbol{G}^{\dagger} \boldsymbol{v}_{R}=0$ to the optimization problem in (6). To this end, due to the separability of the problem in (6), the optimization problem could be separated into two simpler problems as follows ${ }^{3}$

$$
\begin{array}{cc}
\boldsymbol{w}^{\star}=\underset{\substack{\boldsymbol{u}_{R}, \boldsymbol{u}_{T} \\
\text { s.t. }}}{\arg \max } & \left\|\left.\boldsymbol{u}_{R}^{\dagger} \boldsymbol{H}_{1} \boldsymbol{u}_{T}\right|^{2}=\right\| \boldsymbol{u}_{T} \|=1
\end{array}
$$

and

$$
\begin{gathered}
\boldsymbol{w}^{\star}=\underset{\substack{\boldsymbol{v}_{T}, \boldsymbol{v}_{R} \\
\text { s. t. }}}{\arg \max } \\
\boldsymbol{G}^{\dagger} \boldsymbol{v}_{R}=0 \&\left\|\boldsymbol{v}_{T}\right\|=\left\|\boldsymbol{v}_{R}\right\|=1
\end{gathered}
$$

It is to be emphasized that the ZF scheme is optimal in the high SNR region and/or in the presence of strong co-channel interferers (i.e., the asymptotic results of the $\mathrm{ZF}$ scheme are optimal). In order to maximize the harvested energy and information signal power in (7) so as to maximize the overall SINR, the principle of maximum ratio combining (MRC) is used, i.e., the dot product between the two vectors $\boldsymbol{u}_{R}$ and $\boldsymbol{H}_{1} \boldsymbol{u}_{T}$ is maximized iff $\boldsymbol{u}_{R}=\frac{\boldsymbol{H}_{1} \boldsymbol{u}_{T}}{\left\|\boldsymbol{H}_{1} \boldsymbol{u}_{T}\right\|}$. Therefore, the constraint problem in (7) simplifies to [19], [20]

$$
\begin{array}{cl}
\boldsymbol{u}_{T}^{\star}=\underset{\boldsymbol{u}_{T}}{\arg \max } & \left\|\boldsymbol{H}_{1} \boldsymbol{u}_{T}\right\|^{2} \\
\text { s.t. } & \left\|\boldsymbol{u}_{T}\right\|=1
\end{array} .
$$

This is a well known problem (known as the squared spectral norm [19, Eq. (8)]) and its optimal solution is the right or left singular vector of the channel matrix $\boldsymbol{H}_{1}^{\dagger} \boldsymbol{H}_{1}$ corresponding to the strongest eigenmode, i.e., $\boldsymbol{u}_{T}^{\star}=\boldsymbol{u}_{\max }\left(\boldsymbol{H}_{1}^{\dagger} \boldsymbol{H}_{1}\right)$, the eigenvector corresponding to $\lambda_{\max }\left(\boldsymbol{H}_{1}^{\dagger} \boldsymbol{H}_{1}\right)$, the largest eigenvalue of the Wishart matrix $\boldsymbol{H}_{1}^{\dagger} \boldsymbol{H}_{1}$, where $\left\|\boldsymbol{H}_{1} \boldsymbol{u}_{T}\right\|^{2}=$ $\lambda_{\max }\left(\boldsymbol{H}_{1}^{\dagger} \boldsymbol{H}_{1}\right)$.

Similarly, the optimal solution to the constraint optimization problem in (8) is derived as

$$
\boldsymbol{v}_{T}^{\star}=\boldsymbol{v}_{\max }\left(\boldsymbol{H}_{2}^{\dagger} \boldsymbol{P} \boldsymbol{H}_{2}\right)
$$

where $\boldsymbol{P}=\boldsymbol{I}_{N_{D}}-\boldsymbol{G}\left(\boldsymbol{G}^{\dagger} \boldsymbol{G}\right)^{-1} \boldsymbol{G}^{\dagger}$ and $\boldsymbol{v}_{\max }\left(\boldsymbol{H}_{2}^{\dagger} \boldsymbol{P} \boldsymbol{H}_{2}\right)$ is the eigenvector corresponding to $\lambda_{\max }\left(\boldsymbol{H}_{2}^{\dagger} \boldsymbol{P} \boldsymbol{H}_{2}\right)$, the largest eigenvalue of the matrix $\boldsymbol{H}_{2}^{\dagger} \boldsymbol{P} \boldsymbol{H}_{2}$, where $\left\|\boldsymbol{P} \boldsymbol{H}_{2} \boldsymbol{v}_{T}^{\star}\right\|^{2}=\lambda_{\max }\left(\boldsymbol{H}_{2}^{\dagger} \boldsymbol{P} \boldsymbol{H}_{2}\right)$.

Proof: The proof is given in Appendix A.

Therefore, the overall SINR for the time-switching receiver is simplified to

$$
\gamma=\frac{\gamma_{1} \gamma_{2}}{\gamma_{1}+\gamma_{2}+1}
$$

where $\gamma_{1}=\overline{\gamma_{1}}\left\|\boldsymbol{H}_{1}\right\|_{2}^{2}$ is the first-hop SNR, with $\overline{\gamma_{1}}=\frac{\rho}{d_{1}^{\tau}}, \rho=$ $\frac{P_{S}}{\sigma^{2}},\left\|\boldsymbol{H}_{1}\right\|_{2}^{2}=\lambda_{\max }\left(\boldsymbol{H}_{1}^{\dagger} \boldsymbol{H}_{1}\right), \gamma_{2}=\overline{\gamma_{2}}\left\|\boldsymbol{H}_{1}\right\|_{2}^{2}\left\|\boldsymbol{P} \boldsymbol{H}_{2}\right\|_{2}^{2}$ is the second-hop SNR, with $\overline{\gamma_{2}}=\eta \frac{2 \alpha}{(1-\alpha)} \frac{\rho}{d_{1}^{\tau} d_{2}^{\tau}}$, and $\left\|\boldsymbol{P} \boldsymbol{H}_{2}\right\|_{2}^{2}=$

\footnotetext{
${ }^{3}$ Note that maximizing $\left\|\boldsymbol{H}_{1} \boldsymbol{u}_{T}\right\|^{2}$ is exactly the same as maximizing $\left|\boldsymbol{u}_{R}^{\dagger} \boldsymbol{H}_{1} \boldsymbol{u}_{T}\right|^{2}$, i.e., $\underset{\boldsymbol{u}_{T}}{\arg \max }\left\|\boldsymbol{H}_{1} \boldsymbol{u}_{T}\right\|^{2}=\underset{\boldsymbol{u}_{T}}{\arg \max }\left|\boldsymbol{u}_{R}^{\dagger} \boldsymbol{H}_{1} \boldsymbol{u}_{T}\right|^{2}$ [19].
} 


$$
\gamma=\frac{\eta \frac{\rho}{d_{1}^{\tau}} \frac{2 \alpha}{1-\alpha} \frac{\rho}{d_{1}^{\tau} d_{2}^{\tau}}\left|\boldsymbol{u}_{R}^{\dagger} \boldsymbol{H}_{1} \boldsymbol{u}_{T}\right|^{2}\left\|\boldsymbol{H}_{1} \boldsymbol{u}_{T}\right\|^{2}\left|\boldsymbol{v}_{R}^{\dagger} \boldsymbol{H}_{2} \boldsymbol{v}_{T}\right|^{2}}{\frac{\rho}{d_{1}^{\tau}}\left|\boldsymbol{u}_{R}^{\dagger} \boldsymbol{H}_{1} \boldsymbol{u}_{T}\right|^{2}+\eta \frac{2 \alpha}{1-\alpha} \frac{\rho}{d_{1}^{\tau} d_{2}^{\tau}}\left\|\boldsymbol{H}_{1} \boldsymbol{u}_{T}\right\|^{2}\left|\boldsymbol{v}_{R}^{\dagger} \boldsymbol{H}_{2} \boldsymbol{v}_{T}\right|^{2}+\frac{1}{\sigma^{2}}\left\|P_{I}^{\frac{1}{2}} \boldsymbol{G}^{\dagger} \boldsymbol{v}_{R}\right\|^{2}\left(\frac{\rho}{d_{1}^{\tau}}\left|\boldsymbol{u}_{R}^{\dagger} \boldsymbol{H}_{1} \boldsymbol{u}_{T}\right|^{2}+1\right)+1}
$$

$\lambda_{\max }\left(\boldsymbol{H}_{2}^{\dagger} \boldsymbol{P} \boldsymbol{H}_{2}\right)$. The matrix $\boldsymbol{P}$ is an idempotent orthogonal projection matrix, which is used to eliminate the CCI channel at the destination. The term $\left\|\boldsymbol{P} \boldsymbol{H}_{2}\right\|_{2}^{2}$ is the largest eigenvalue of the Wishart matrix $\boldsymbol{H}_{2}^{\dagger} \boldsymbol{P} \boldsymbol{H}_{2}$ with dimensions $\left(N_{D}-M\right) \times$ $N_{R}$ [21, Eq. (37)].

It is to be emphasized that analyzing the performance of the overall SINR in (11) is much more complicated than that of the conventional self-powered MIMO relaying systems ${ }^{4}$ due to the dependency of the second-hop SNR on the first-hop SNR (i.e., $\gamma_{2}=\frac{2 \alpha}{(1-\alpha)} \frac{\eta}{d_{2}^{\tau}}\left\|\boldsymbol{P} \boldsymbol{H}_{2}\right\|_{2}^{2} \gamma_{1}$ ). Therefore, the presence of high correlation between the first-hop and second-hop SNRs and the complicated statistics of the second-hop SNR (i.e., $\gamma_{2}=\overline{\gamma_{2}}\left\|\boldsymbol{H}_{1}\right\|_{2}^{2}\left\|\boldsymbol{P} \boldsymbol{H}_{2}\right\|_{2}^{2}$ ) make the analysis of such systems a mathematically challenging problem.

\section{B. Power-Splitting Receiver (PSR)}

The power-splitting receiver for information processing and energy harvesting is shown in Fig. 1(c), where $\beta$ with $0<$ $\beta<1$ is the fraction of received power which the relay uses for energy harvesting during the first half of the block time. The remaining power fraction $(1-\beta)$ is used for the $\boldsymbol{S} \rightarrow \boldsymbol{R}$ information transmission during the first half of the block time. All energy scavenged from the RF signal during the first half of the block time is consumed by the energy-constrained relay when forwarding the source message from the $R \rightarrow D$ during the second half of the block time. It is clear that the choice of the power fraction $\beta$ affects the overall achievable throughput. Therefore, the optimal choice for the power fraction $\beta$ which maximizes the achievable throughput is selected.

Using the same transmit and receive precoding vectors derived in (6), the received signal at the energy harvesting receiver after energy MRT beamforming is given by

$$
\boldsymbol{y}_{E}=\sqrt{\frac{\beta P_{S}}{d_{1}^{\tau}}} \boldsymbol{H}_{1} \boldsymbol{u}_{T}^{\star} x_{E}+\boldsymbol{n}_{R} .
$$

Therefore, the relay transmit power after $T / 2$ harvesting time is given by $P_{R}=\frac{\eta\left(\beta \frac{P_{S}}{d_{1}^{\tau}}\left\|\boldsymbol{H}_{1}\right\|_{2}^{2}\right) T / 2}{T / 2}=\eta \beta \frac{P_{S}}{d_{1}^{\tau}}\left\|\boldsymbol{H}_{1}\right\|_{2}^{2}$.

Accordingly, after the power-splitting receiver, the MRC combined received information signal at the relay, after MRT beamforming at the transmitter, is given as

$$
y_{R}=\sqrt{\frac{(1-\beta) P_{S}}{d_{1}^{\tau}}}\left\|\boldsymbol{H}_{1}\right\|_{2} x_{S}+\boldsymbol{u}_{R}^{\star \dagger} \boldsymbol{n}_{R} .
$$

\footnotetext{
${ }^{4}$ The overall SINR for the wirelessly powered MIMO relaying systems in (11) is written as $\gamma=\frac{\overline{\gamma_{1}}\left\|\boldsymbol{H}_{1}\right\|_{2}^{2} \overline{\gamma_{2}}\left\|\boldsymbol{H}_{1}\right\|_{2}^{2}\left\|\boldsymbol{P} \boldsymbol{H}_{2}\right\|_{2}^{2}}{\overline{\gamma_{1}}\left\|\boldsymbol{H}_{1}\right\|_{2}^{2}+\overline{\gamma_{2}}\left\|\boldsymbol{H}_{1}\right\|_{2}^{2}\left\|\boldsymbol{P} \boldsymbol{H}_{2}\right\|_{2}^{2}+1}$. Note that the overall SNR for the conventional self-powered MIMO relaying systems is given by [22] $\gamma=\frac{\rho_{1}\left\|\boldsymbol{H}_{1}\right\|_{2}^{2} \rho_{2}\left\|\boldsymbol{H}_{2}\right\|_{2}^{2}}{\rho_{1}\left\|\boldsymbol{H}_{1}\right\|_{2}^{2}+\rho_{2}\left\|\boldsymbol{H}_{2}\right\|_{2}^{2}+1}$, where $\rho_{1}$ is the source average transmit SNR and $\rho_{2}$ is the relay average transmit SNR.
}

The $\mathrm{ZF}$ combined received signal at the destination is expressed as

$$
y_{D}=\sqrt{\frac{P_{R}}{d_{2}^{\tau}}} \mathcal{G}\left\|\boldsymbol{P} \boldsymbol{H}_{2}\right\|_{2} y_{R}+\boldsymbol{v}_{R}^{\star \dagger} \boldsymbol{n}_{D} .
$$

Note that the channel assisted relay normalizing constant $\mathcal{G}$ in the power-splitting receiver is given by

$$
\mathcal{G}^{-1}=\sqrt{\frac{(1-\beta) P_{S}}{d_{1}^{\tau}}\left\|\boldsymbol{H}_{1}\right\|_{2}^{2}+\sigma^{2}} .
$$

Therefore, the overall SINR for the power-splitting receiver is simplified to

$$
\gamma=\frac{\gamma_{1} \gamma_{2}}{\gamma_{1}+\gamma_{2}+1}
$$

where $\gamma_{1}=\overline{\gamma_{1}}\left\|\boldsymbol{H}_{1}\right\|_{2}^{2}$ is the first-hop SNR, with $\overline{\gamma_{1}}=\frac{(1-\beta) \rho}{d_{1}^{\tau}}$, and $\gamma_{2}=\overline{\gamma_{2}}\left\|\boldsymbol{H}_{1}\right\|_{2}^{2}\left\|\boldsymbol{P} \boldsymbol{H}_{2}\right\|_{2}^{2}$ is the second-hop SNR, with $\overline{\gamma_{2}}=\eta \beta \frac{\rho}{d_{1}^{\tau} d_{2}^{\tau}}$.

\section{Performance AnAlysis}

The performance analysis of the overall SINR for MIMO wireless information and power transfer systems in (11) for TSR and in (16) for PSR is a challenging mathematical problem due to the presence of high correlation between the first-hop and second-hop SNRs and the complicated statistics of the second-hop SNR. Therefore, in order to simplify the mathematical tractability and attain closed-form expressions so as to gain a better insights onto the performance analysis of the system, the overall SINR in (11) and (16) can be tightly upper-bounded by ${ }^{5}$

$$
\gamma \leq \gamma^{\text {up }}=\frac{\gamma_{1} \gamma_{2}}{\gamma_{1}+\gamma_{2}}
$$

Therefore, the overall SINR upper-bound can be simplified to

$$
\gamma^{\mathrm{up}}=\frac{\overline{\gamma_{1} \gamma_{2}}\left\|\boldsymbol{H}_{1}\right\|_{2}^{2}\left\|\boldsymbol{P} \boldsymbol{H}_{2}\right\|_{2}^{2}}{\overline{\gamma_{2}}\left\|\boldsymbol{P} \boldsymbol{H}_{2}\right\|_{2}^{2}+\overline{\gamma_{1}}} .
$$

It is to be emphasized that the asymptotic results of this bound are exact. Hence, the throughput performance of the time-switching receiver and power-splitting receiver are analyzed for delay-limited and delay-tolerant transmission modes, namely, exact outage probability as well as tight approximate and lower-bound ergodic capacity expressions for the proposed overall SINR upper-bound are derived in closed-form.

\footnotetext{
${ }^{5}$ It is well known in the conventional self-powered half-duplex relaying literature that the overall SNR $\frac{\gamma_{1} \gamma_{2}}{\gamma_{1}+\gamma_{2}+1}$ can be tightly upper-bounded by $\frac{\gamma_{1} \gamma_{2}}{\gamma_{1}+\gamma_{2}}$ (see e.g., [23, Eq. (6)]). In addition, the upper-bound $\frac{\gamma_{1} \gamma_{2}}{\gamma_{1}+\gamma_{2}}$ can be further upper-bounded by $\min \left(\gamma_{1}, \gamma_{2}\right)$ (see e.g., [24, Eq. (8)]).
} 


\section{A. Outage Probability Analysis}

In this section, the SINR outage probability of the overall SINR of MIMO energy-constrained relaying system is investigated. The SINR outage probability is defined as the probability that the instantaneous overall SINR $\gamma$, falls below a pre-defined SINR threshold $\gamma_{T}{ }^{6}$, which can be given as

$$
\begin{aligned}
\mathrm{P}_{\text {out }}\left(\gamma_{T}\right) & =\operatorname{Pr}\left(\gamma<\gamma_{T}\right) \\
& =F_{\gamma}\left(\gamma_{T}\right)
\end{aligned}
$$

where $F_{\gamma}(\cdot)$ denotes the cumulative distribution function (CDF) of the overall SINR.

In this section, closed-form expression for the outage probability of the overall SINR upper-bound (lower-bound outage probability expression for the exact overall SINR) is derived. The outage probability of the overall SINR upper-bound is given as

$$
F_{\gamma^{\text {up }}}\left(\gamma_{T}\right)=\operatorname{Pr}\left(\frac{\overline{\gamma_{1} \gamma_{2}}\left\|\boldsymbol{H}_{1}\right\|_{2}^{2}\left\|\boldsymbol{P} \boldsymbol{H}_{2}\right\|_{2}^{2}}{\overline{\gamma_{2}}\left\|\boldsymbol{P} \boldsymbol{H}_{2}\right\|_{2}^{2}+\overline{\gamma_{1}}}<\gamma_{T}\right) .
$$

Note that the distributions of $\left\|\boldsymbol{H}_{1}\right\|^{2}$ and $\left\|\boldsymbol{P} \boldsymbol{H}_{2}\right\|^{2}$ follow Wishart distribution with dimensions $N_{R} \times N_{S}$ and $\left(N_{D}-M\right) \times N_{R}$ degrees of freedom, respectively. Hence, the cumulative distribution function (CDF) and probability density function (PDF) of $\left\|\boldsymbol{H}_{1}\right\|_{2}^{2}$ and $\left\|\boldsymbol{P} \boldsymbol{H}_{2}\right\|_{2}^{2}$, respectively, are given as [19]

$$
\begin{aligned}
& F_{\left\|\boldsymbol{H}_{1}\right\|_{2}^{2}}(x)= \\
& 1-\sum_{i=1}^{\min \left(N_{S}, N_{R}\right)} \sum_{j=\left|N_{S}-N_{R}\right|}^{\left(N_{S}+N_{R}\right) i-2 i^{2}} \sum_{k=0}^{j} \frac{i^{k} d_{1}(i, j)}{k !} x^{k} e^{-i x}
\end{aligned}
$$

and

$$
\begin{aligned}
& f_{\left\|\boldsymbol{P} \boldsymbol{H}_{2}\right\|_{2}^{2}}(y)= \\
& \sum_{l=1}^{\min \left(N_{R}, N_{D}-M\right)} \sum_{m=\left|N_{R}-N_{D}+M\right|}^{\left(N_{R}+N_{D}-M\right) l-2 l^{2}} \frac{l^{m+1} d_{2}(l, m)}{m !} y^{m} e^{-l y}
\end{aligned}
$$

where the coefficients $d_{l}(a, b), l=1,2$ are given in [19] for some system configurations, and they can be efficiently evaluated using [20, Algorithm 1].

Theorem 1. A closed-form lower-bound outage probability expression of the overall SINR for MIMO energy-constrained relaying systems with information and energy beamforming can be expressed as in (23), shown at the top of next page, where $\mathcal{K}_{v}(z)$ is the modified bessel function of the second kind of order $v$.

Proof: The proof is given in Appendix B.

Corollary 2. In the case of energy-constrained single antenna relay (i.e., $N_{R}=1$ ), the closed-form lower-bound outage

\footnotetext{
${ }^{6}$ Note that in contrast to (19), the information outage probability is defined as the probability that the instantaneous mutual information, $\mathcal{I}=$ $\frac{1}{2} \log _{2}(1+\gamma)$, falls below a target rate of $R_{0}$ bits per channel use. Note that the pre-defined SINR threshold may be given as $\gamma_{T}=2^{2 R_{0}}-1$.
}

probability expression is simplified to [25]

$$
\begin{gathered}
F_{\gamma^{\text {up }}}\left(\gamma_{T}\right)=1-\frac{2 e^{-\frac{\gamma_{T}}{\gamma_{1}}}}{\Gamma\left(N_{D}-M\right)} \sum_{k=0}^{N_{S}-1} \frac{1}{k !}\left(\frac{\gamma_{T}}{\overline{\gamma_{1}}}\right)^{k} \sum_{n=0}^{k}\left(\begin{array}{c}
k \\
n
\end{array}\right) \\
\times\left(\frac{\overline{\gamma_{1}}}{\overline{\gamma_{2}}}\right)^{n}\left(\frac{\gamma_{T}}{\overline{\gamma_{2}}}\right)^{\frac{N_{D}-M-n}{2}} \mathcal{K}_{N_{D}-M-n}\left(2 \sqrt{\frac{\gamma_{T}}{\overline{\gamma_{2}}}}\right) \cdot \quad
\end{gathered}
$$

In the case of delay-constrained (limited) (DL) transmission, the source transmits information at a fixed rate $R_{0}=$ $\log _{2}\left(1+\gamma_{T}\right)$ bits/sec/Hz. Therefore, the average throughput $\mathcal{R}$ is given as [4]

$$
\mathcal{R}^{\mathrm{DL}}=\frac{(1-\alpha)}{2}\left(1-\mathrm{P}_{\text {out }}\left(\gamma_{T}\right)\right) \log _{2}\left(1+\gamma_{T}\right)
$$

for the time-switching receiver, and

$$
\mathcal{R}^{\mathrm{DL}}=\frac{1}{2}\left(1-\mathrm{P}_{\text {out }}\left(\gamma_{T}\right)\right) \log _{2}\left(1+\gamma_{T}\right)
$$

for the power-splitting receiver.

1) Asymptotic analysis: Note that though theorem 1 provides an efficient means for analyzing the outage probability of the system, this formula is quite complicated as it does not provide simple insights onto the performance analysis of the system. Therefore, to validate and characterize the achievable diversity order of MIMO energy-constrained relaying systems with information and energy beamforming, the overall SINR is upper-bounded to

$$
\gamma \leq \gamma^{\mathrm{up} 2}=\min \left(\gamma_{1}, \gamma_{2}\right)
$$

Therefore, the asymptotic outage probability of MIMO energy-constrained relaying systems is derived as

$$
\begin{aligned}
F_{\gamma}^{\infty}\left(\gamma_{T}\right)= & \frac{\prod_{l=0}^{s_{1}-1} l !}{\prod_{l=0}^{s_{1}-1}\left(t_{1}+l\right) !}\left(\frac{\gamma_{T}}{\overline{\gamma_{1}}}\right)^{N_{S} N_{R}} \\
& +\frac{\prod_{l=0}^{s_{2}-1} l !}{\prod_{l=0}^{s_{2}-1}\left(t_{2}+l\right) !}\left(\frac{\gamma_{T}}{\overline{\gamma_{2}}}\right)^{N_{R}\left(N_{D}-M\right)} \mathcal{K}
\end{aligned}
$$

where $s_{1}=\min \left(N_{S}, N_{R}\right), t_{1}=\max \left(N_{S}, N_{R}\right), s_{2}=$ $\min \left(N_{R}, N_{D}-M\right), t_{2}=\max \left(N_{R}, N_{D}-M\right)$, and $\mathcal{K}$ is given as

$$
\begin{aligned}
\mathcal{K}=\sum_{i=1}^{s_{1}} \sum_{j=\left|N_{S}-N_{R}\right|}^{\left(N_{S}+N_{R}\right) i-2 i^{2}} \frac{d_{1}(i, j)}{j !} i^{N_{R}\left(N_{D}-M\right)} & \\
& \times \Gamma\left(j-N_{R}\left(N_{D}-M\right)+1, i \frac{\gamma_{T}}{\overline{\gamma_{1}}}\right) .
\end{aligned}
$$

Proof: The proof is given in Appendix C.

It is straight forward to show from (28) that the achievable diversity order of MIMO relaying system with information and energy beamforming is $\min \left(N_{S} N_{R}, N_{R}\left(N_{D}-M\right)\right)$. It is to be emphasized that $M$ degrees of freedom is used for null space projection to eliminate the CCI. Note that in contrast to the conventional MIMO self-powered relaying systems, the asymptotic outage probability of MIMO energy-constrained relaying system with information and energy beamforming decays slower due to the term $\mathcal{K}$ in (28) which is not present 


$$
\begin{array}{r}
F_{\gamma^{\text {up }}}\left(\gamma_{T}\right)=1-2 \sum_{i=1}^{\min \left(N_{S}, N_{R}\right)} \sum_{j=\left|N_{S}-N_{R}\right|}^{\left(N_{S}+N_{R}\right) i-2 i^{2}} \sum_{k=0}^{j} \frac{i^{k} d_{1}(i, j)}{k !}\left(\frac{\gamma_{T}}{\overline{\gamma_{1}}}\right)^{k} e^{-\frac{i \gamma_{T}}{\bar{\gamma}_{1}}} \sum_{l=1}^{\min \left(N_{R}, N_{D}-M\right)} \sum_{m=\left|N_{R}-N_{D}+M\right|}^{\left(N_{R}+N_{D}-M\right) l-2 l^{2}} \\
\times \frac{l^{m+1} d_{2}(l, m)}{m !} \sum_{n=0}^{k}\left(\begin{array}{l}
k \\
n
\end{array}\right)\left(\frac{\overline{\gamma_{1}}}{\overline{\gamma_{2}}}\right)^{n}\left(\frac{i \gamma_{T}}{l \overline{\gamma_{2}}}\right)^{\frac{m-n+1}{2}} \mathcal{K}_{m-n+1}\left(2 \sqrt{\frac{i l \gamma_{T}}{\overline{\gamma_{2}}}}\right)
\end{array}
$$

in the self-powered relaying systems ${ }^{7}$. This implies that the convergence of $F_{\gamma}^{\infty}\left(\gamma_{T}\right)$ in wireless information and power transfer systems is exceedingly slower compared to that of self-powered relaying systems.

\section{B. Ergodic Capacity Analysis}

The capacity analysis is an important performance measure for any wireless communication system as it results in the maximum achievable rate. In the delay-tolerant transmission, the throughput is defined by analyzing the ergodic capacity. Here, in contrast to the delay-limited transmission mode, where the source transmits at a constant rate $R_{0}$ in order to meet the outage constraint, the source can transmit data at any rate upper bounded by the ergodic capacity. The ergodic capacity is defined as the expected value of the instantaneous mutual information between the source and destination, written as

$$
C=\frac{1}{2} \mathbb{E}\left[\log _{2}(1+\gamma)\right]
$$

Unfortunately, the exact analysis of the ergodic capacity in (30) is mathematically intractable. Therefore, the ergodic capacity is evaluated for the proposed overall SINR upperbound as follows

$$
C^{\text {up }}=\frac{1}{2} \mathbb{E}\left[\log _{2}\left(1+\frac{\overline{\gamma_{1} \gamma_{2}}\left\|\boldsymbol{H}_{1}\right\|_{2}^{2}\left\|\boldsymbol{P} \boldsymbol{H}_{2}\right\|_{2}^{2}}{\overline{\gamma_{2}}\left\|\boldsymbol{P} \boldsymbol{H}_{2}\right\|_{2}^{2}+\overline{\gamma_{1}}}\right)\right] .
$$

An exact closed-form expression for the ergodic capacity (31) is still a challenging mathematical problem. Therefore, in this section we introduce an approximate as well as a tight lower-bound closed-form expressions for the ergodic capacity of the overall SINR upper-bound (31).

1) Approximate Expression: Here, a closed-form approximate ergodic capacity expression is obtained once exploiting the Taylor series expansion of the function $\log _{2}\left(1+\gamma^{\text {up }}\right)$ around the mean value of the instantaneous overall SINR upper-bound $\mathbb{E}\left(\gamma^{\text {up }}\right)$. Therefore, an approximate ergodic capacity expression can be written as (see e.g., [26, Eq. (6)] and [27, Eq. (26)])

$C^{\mathrm{up}} \approx \frac{1}{2 \ln 2}\left(\ln \left(1+\mathbb{E}\left(\gamma^{\mathrm{up}}\right)\right)-\frac{\mathbb{E}\left(\left(\gamma^{\mathrm{up}}\right)^{2}\right)-\mathbb{E}\left(\gamma^{\mathrm{up}}\right)^{2}}{2\left(1+\mathbb{E}\left(\gamma^{\mathrm{up}}\right)\right)^{2}}\right)$

\footnotetext{
${ }^{7}$ Note that in the conventional self-powered relaying systems, the $\boldsymbol{S} \rightarrow \boldsymbol{R}$ and $\boldsymbol{R} \rightarrow \boldsymbol{D}$ links are independent. However, in wireless information and power transfer relaying systems with energy-constrained relay, the $\boldsymbol{R} \rightarrow \boldsymbol{D}$ link highly depends on the $\boldsymbol{S} \rightarrow \boldsymbol{R}$ link $\left(\gamma_{1}\right)$ through which the harvested energy propagate.
}

Note that in order to evaluate the approximate ergodic capacity expression in (32), moments of the overall SINR upper-bound are required. Therefore, next we derive a closedform expression for the moments of the overall SINR upperbound.

Theorem 3. A closed-form expression for the moments of the overall SINR upper-bound for MIMO energy-constrained relaying systems with information and energy beamforming is derived as in (33), shown at the top of next page, where $\Psi(\alpha, \gamma ; z)$ is the Tricomi confluent hypergeometric function of the second kind.

Proof: The proof is given in Appendix D.

Corollary 4. In the case of energy-constrained single antenna relay (i.e., $N_{R}=1$ ), the closed-form expression for the moments of the overall SINR upper-bound is simplified to [25]

$$
\begin{aligned}
\mathbb{E}\left[\left(\gamma^{\text {up }}\right)^{k}\right]={\overline{\gamma_{1}}}^{k} \frac{\Gamma\left(N_{S}+k\right) \Gamma\left(N_{D}-M+k\right)}{\Gamma\left(N_{S}\right) \Gamma\left(N_{D}-M\right)} \\
\times \Psi\left(k, 1-N_{D}+M ; \frac{\overline{\gamma_{1}}}{\overline{\gamma_{2}}}\right) .
\end{aligned}
$$

2) Lower-Bound Expression: In order to find a closed-form lower-bound expression for the ergodic capacity in (31), we take advantage of the fact that $\log _{2}(1+\alpha \exp (x))$ is a convex function in $x$ for $\alpha>0$. Therefore, equation (31) can be tightly lower-bounded by

$$
\begin{aligned}
& C^{\text {up }} \geq \\
& \frac{1}{2} \log _{2}\left(1+\exp \left(\mathbb{E}\left\{\ln \left[\frac{\overline{\gamma_{1} \gamma_{2}}\left\|\boldsymbol{H}_{1}\right\|_{2}^{2}\left\|\boldsymbol{P} \boldsymbol{H}_{2}\right\|_{2}^{2}}{\overline{\gamma_{2}}\left\|\boldsymbol{P} \boldsymbol{H}_{2}\right\|_{2}^{2}+\overline{\gamma_{1}}}\right]\right\}\right)\right) .
\end{aligned}
$$

Theorem 5. A closed-form lower-bound expression for the ergodic capacity of the overall SINR upper-bound for MIMO energy-constrained relaying systems with information and energy beamforming is expressed as in (36), shown at the top of next page, where

$$
\begin{aligned}
\mathbb{E}\left[\ln \left(\left\|\boldsymbol{H}_{1}\right\|_{2}^{2}\right)\right]= \\
\sum_{i=1}^{s_{1}} \sum_{j=\left|N_{S}-N_{R}\right|}^{\left(N_{S}+N_{R}\right) i-2 i^{2}} d_{1}(i, j)[\psi(j+1)-\ln i]
\end{aligned}
$$




$$
\begin{aligned}
& \mathbb{E}\left[\left(\gamma^{\mathrm{up}}\right)^{k}\right]=\bar{\gamma}_{1} \sum_{i=1}^{\min \left(N_{S}, N_{R}\right)} \sum_{j=\left|N_{S}-N_{R}\right|}^{\left(N_{S}+N_{R}\right) i-2 i^{2}} \frac{d_{1}(i, j)}{j !}\left(\frac{1}{i}\right)^{k} \Gamma(k+j+1) \sum_{l=1}^{\min \left(N_{R}, N_{D}-M\right)} \sum_{m=\left|N_{R}-N_{D}+M\right|}^{\left(N_{R}+N_{D}-M\right) l-2 l^{2}} \frac{d_{2}(l, m)}{m !} \\
& \times \Gamma(k+m+1) \Psi\left(k,-m ; \frac{l \overline{\gamma_{1}}}{\overline{\gamma_{2}}}\right)
\end{aligned}
$$

$$
C^{\mathrm{up}} \geq \frac{1}{2} \log _{2}\left(1+\exp \left(\mathbb{E}\left[\ln \left(\left\|\boldsymbol{H}_{1}\right\|_{2}^{2}\right)\right]+\mathbb{E}\left[\ln \left(\overline{\gamma_{2}}\left\|\boldsymbol{P} \boldsymbol{H}_{2}\right\|_{2}^{2}\right)\right]-\mathbb{E}\left[\ln \left(1+\frac{\overline{\gamma_{2}}}{\overline{\gamma_{1}}}\left\|\boldsymbol{P} \boldsymbol{H}_{2}\right\|_{2}^{2}\right)\right]\right)\right)
$$

and

$$
\begin{aligned}
\mathbb{E}\left[\ln \left(\overline{\gamma_{2}}\left\|\boldsymbol{P} \boldsymbol{H}_{2}\right\|_{2}^{2}\right)\right]=\ln \left(\overline{\gamma_{2}}\right)+ \\
\sum_{l=1}^{s_{2}} \sum_{m=\left|N_{R}-N_{D}+M\right|}^{\left(N_{R}+N_{D}-M\right) l-2 l^{2}} d_{2}(l, m)[\psi(m+1)-\ln l]
\end{aligned}
$$

and

$$
\begin{aligned}
& \mathbb{E}\left[\ln \left(1+\frac{\overline{\gamma_{2}}}{\overline{\gamma_{1}}}\left\|\boldsymbol{P} \boldsymbol{H}_{2}\right\|_{2}^{2}\right)\right]= \\
& \sum_{l=1}^{s_{2}} \sum_{m=\left|N_{R}-N_{D}+M\right|}^{\left(N_{R}+N_{D}-M\right) l-2 l^{2}} \sum_{n=0}^{m} d_{2}(l, m) e^{\frac{l \overline{\gamma_{1}}}{\bar{\gamma}_{2}}} \mathrm{E}_{1+n}\left(\frac{l \overline{\gamma_{1}}}{\overline{\gamma_{2}}}\right)
\end{aligned}
$$

where $\psi(\cdot)$ is the digamma (psi) function [28, Eq. (8.360.1)], and $\mathrm{E}_{n}(\cdot)$ is the exponential integral function [29, Eq. (5.1.4)].

Proof: The proof is given in Appendix E.

Corollary 6. In the case of energy-constrained single antenna relay (i.e., $N_{R}=1$ ), the closed-form lower-bound expression for the ergodic capacity of the overall SINR upper-bound for MIMO energy-constrained relaying system is simplified to (40) [25], shown at the top of the page.

3) Asymptotic analysis: The ergodic capacity at high SNR can be written as

$$
\begin{aligned}
C^{\infty}=\frac{1}{2} \mathbb{E}\left[\log _{2}\left(\frac{\overline{\gamma_{2}}\left\|\boldsymbol{H}_{1}\right\|_{2}^{2}\left\|\boldsymbol{P} \boldsymbol{H}_{2}\right\|_{2}^{2}}{\overline{\overline{\gamma_{2}}}\left\|\boldsymbol{P} \boldsymbol{H}_{2}\right\|_{2}^{2}+1}\right)\right] \\
=\frac{1}{2} \mathbb{E}\left[\log _{2}\left(\left\|\boldsymbol{H}_{1}\right\|_{2}^{2}\right)\right]+\frac{1}{2} \mathbb{E}\left[\log _{2}\left(\overline{\gamma_{2}}\left\|\boldsymbol{P} \boldsymbol{H}_{2}\right\|_{2}^{2}\right)\right] \\
-\frac{1}{2} \mathbb{E}\left[\log _{2}\left(1+\frac{\overline{\gamma_{2}}}{\overline{\gamma_{1}}}\left\|\boldsymbol{P} \boldsymbol{H}_{2}\right\|_{2}^{2}\right)\right] .
\end{aligned}
$$

In the case of delay-tolerant (DT) transmission, the source transmits information at any rate less than or equal to the ergodic capacity. Therefore, the average throughput $\mathcal{R}$ is given as

$$
\mathcal{R}^{\mathrm{DT}}=(1-\alpha) C
$$

for the time-switching receiver, and

$$
\mathcal{R}^{\mathrm{DT}}=C
$$

for the power-splitting receiver.

The optimal ratio $w^{\star}$ for the time-switching receiver or power-splitting receiver $w \in\{\alpha, \beta\}$ for the delay-limited and

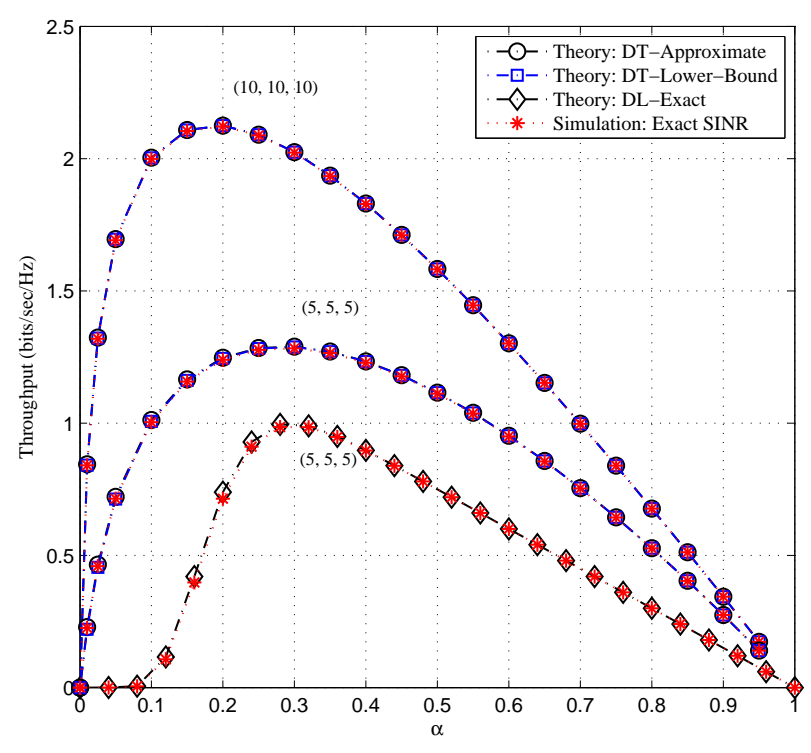

Figure 2: The delay-tolerant (DT) and delay-limited (DL) transmission throughput for TSR against the energy harvesting ratio $\alpha$, with $M=1$ and $\rho=20 \mathrm{~dB}$.

delay-tolerant transmission $z \in\{\mathrm{DL}, \mathrm{DT}\}$ could be evaluated by solving the following optimization problem

$$
\begin{array}{cc}
w^{\star}=\underset{w}{\arg \max } & \left.\left.\mathcal{R}^{z} \text { (in Eq. (25 or } 26 \text { or } 42 \text { or } 43\right)\right) \\
\text { subject to } & 0<w<1
\end{array}
$$

\section{NumericAl RESUlts}

In this section, we analyze and validate the presented theoretical results with Monte Carlo simulations. In addition, the impact of key system parameters on the system throughput are investigated. In the delay limited transmission, the source transmission rate is set to $R_{0}=3 \mathrm{bits} / \mathrm{sec} / \mathrm{Hz}$. Hence, the predefined SINR threshold is given by $\gamma_{T}=2^{R_{0}}-1=7$. Besides, without loss of generality, the energy conversion efficiency is chosen as $\eta=0.8$, the distances $d_{1}$ and $d_{2}$ are set to 5 , and the path loss exponent $\tau=2$. The values of each antenna configurations $N_{S}, N_{R}$, and $N_{D}$ are shown at the figure labels as $\left(N_{S}, N_{R}, N_{D}\right)$ in the case of fixed $M$, and as $\left(N_{S}, N_{R}, N_{D}, M\right)$ in the case of different $M$. 


$$
C^{\text {up }} \geq \frac{1}{2} \log _{2}\left(1+\exp \left(\psi\left(N_{S}\right)+\ln \overline{\gamma_{2}}+\psi\left(N_{D}-M\right)-\sum_{k=0}^{N_{D}-M-1} e^{\frac{\overline{\gamma_{1}}}{\overline{\gamma_{2}}}} \mathrm{E}_{1+k}\left(\frac{\overline{\gamma_{1}}}{\overline{\gamma_{2}}}\right)\right)\right)
$$

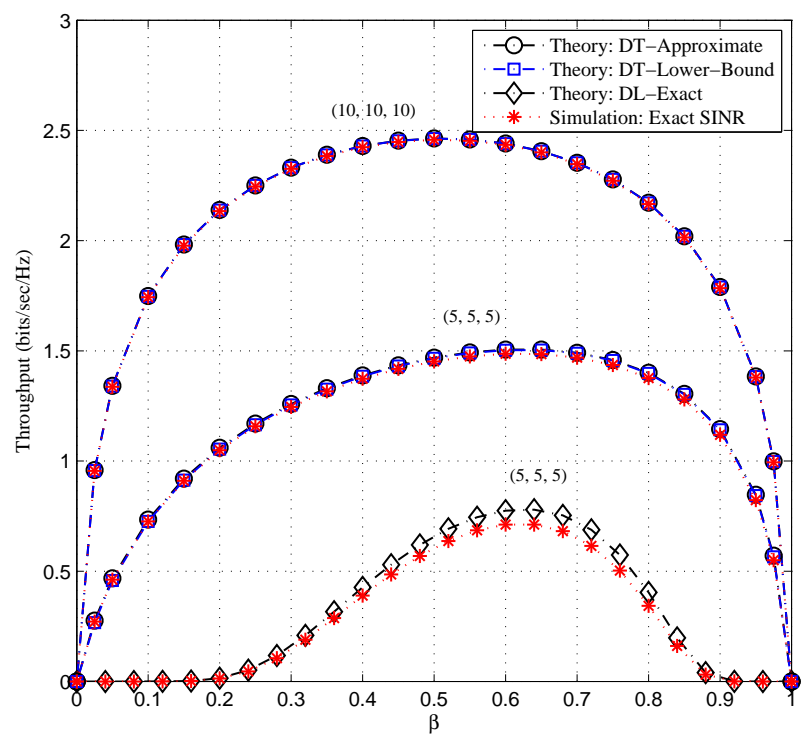

Figure 3: The delay-tolerant (DT) and delay-limited (DL) transmission throughput for PSR against the energy harvesting ratio $\beta$, with $M=1$ and $\rho=20 \mathrm{~dB}$.

In Figs. 2-3, the throughput of delay-tolerant and delaylimited transmission modes versus the energy harvesting ratio $w \in\{\alpha, \beta\}$ is presented for the TSR and PSR, respectively, where the source average $\operatorname{SNR} \rho$ is chosen to be $20 \mathrm{~dB}$, and the number of interferes are set to $M=1$. Monte Carlo simulations for the performance of the exact overall SINR (11) for the TSR and (16) for the PSR are used to validate the new analytical expressions (23) for the outage probability, (32) and (36) for the ergodic capacity, through the average throughput of the delay-limited and delay-tolerant transmission modes (25) and (42) for the TSR, and (26) and (43) for the PSR. It is seen that the simulation and proposed analytical expressions provide an excellent tightness which corroborate the accuracy of the proposed analytical expressions, and become exact at high SNR and/or high diversity order. Besides, due to the mathematical intractability of the problem, the optimal energy harvesting ratio $w^{\star}$ is numerically obtained. It is seen in Figs. 2-3 that the throughput increases as the energy harvesting ratio $w$ increases until an optimum is reached (based on (44)) beyond which it starts to decrease again. This is justified by the trade-off between energy harvesting and information transmission ratios; increasing the time (in the TSR case) or power (in the PSR case) ratio reserved for energy harvesting improves the relay transmission power, however, this results in a reduced overall throughput as less time or power ratio is left for information transmission. Furthermore, the benefit of MRT/MRC information and energy hop-by-hop beamforming

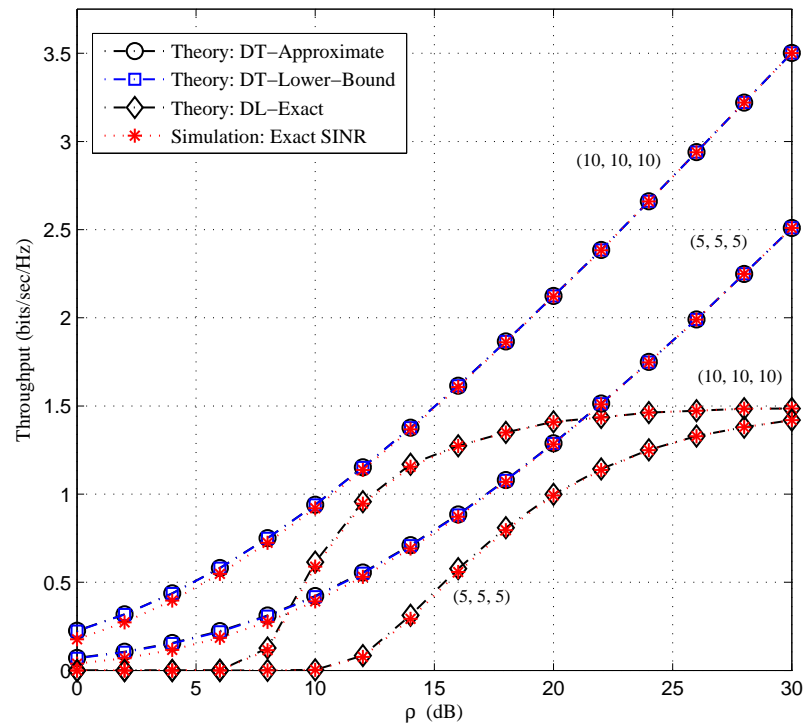

Figure 4: The delay-tolerant (DT) and delay-limited (DL) transmission throughput for the TSR with optimal energy harvesting ratio $\alpha^{\star}$ against the first-hop average $\operatorname{SNR} \rho$, with $M=1$.

are twofold: it results in a substantial improvement in the average throughput of the system as it maximizes the overall SINR as well as the harvested energy, it also reduces the optimal energy harvesting ratio $w^{\star}$, hence, more time (in the TSR case) or power (in the PSR case) is left for information transmission.

In Figs. 4-5, the throughput of DT and DL transmission modes with optimal energy harvesting ratio $w^{\star}$ versus first-hop average SNR $\rho$ is presented for the TSR and PSR, respectively, where the number of interferers are set to $M=1$. The tightness of the proposed closed-form analytical expressions are clearly seen when compared to the exact Monte Carlo simulations. It is seen that the throughput of DL transmission mode is upper bounded by half the transmission rate $R_{0}=3$ bits $/ \mathrm{sec} / \mathrm{Hz}$, where the throughput of DT transmission mode increases without bound as the first-hop average SNR increases. Our results show that multi-antenna nodes provide a significant throughput improvement as compared with single antenna nodes.

Fig. 6 compares the throughput performance of the DT transmission mode for the TSR and PSR, where the approximate closed-form ergodic capacity expression (32) is used to evaluate the DT transmission throughput (42) and (43) for the TSR and PSR, respectively. The achievable diversity order of the configurations $(10,10,10,1)$, $(5,5,5,1),(5,5,5,4)$, and $(3,3,3,2)$, which is given by 


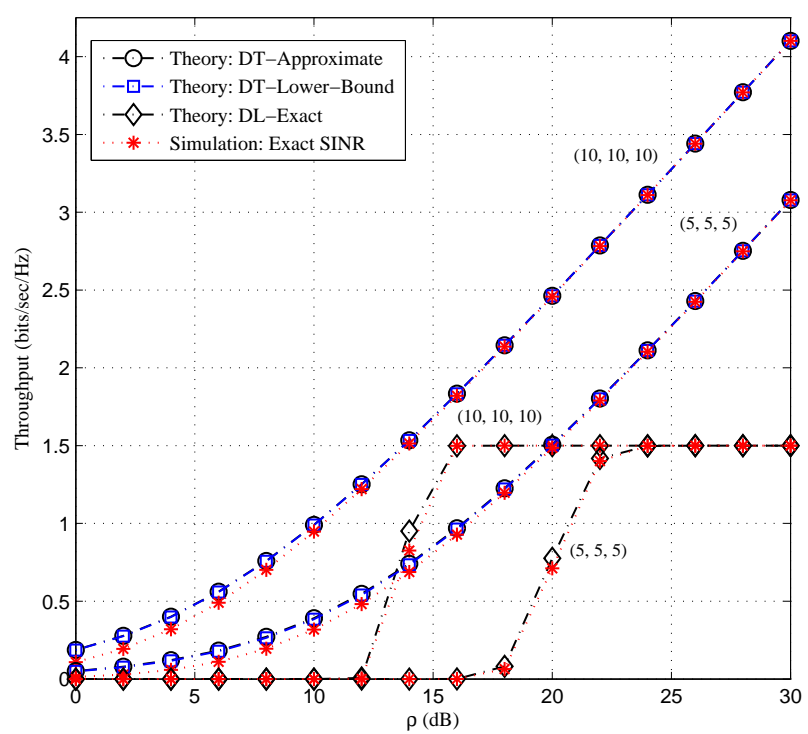

Figure 5: The delay-tolerant (DT) and delay-limited (DL) transmission throughput for the PSR with optimal energy harvesting ratio $\beta^{\star}$ against the first-hop average $\operatorname{SNR} \rho$, with $M=1$.

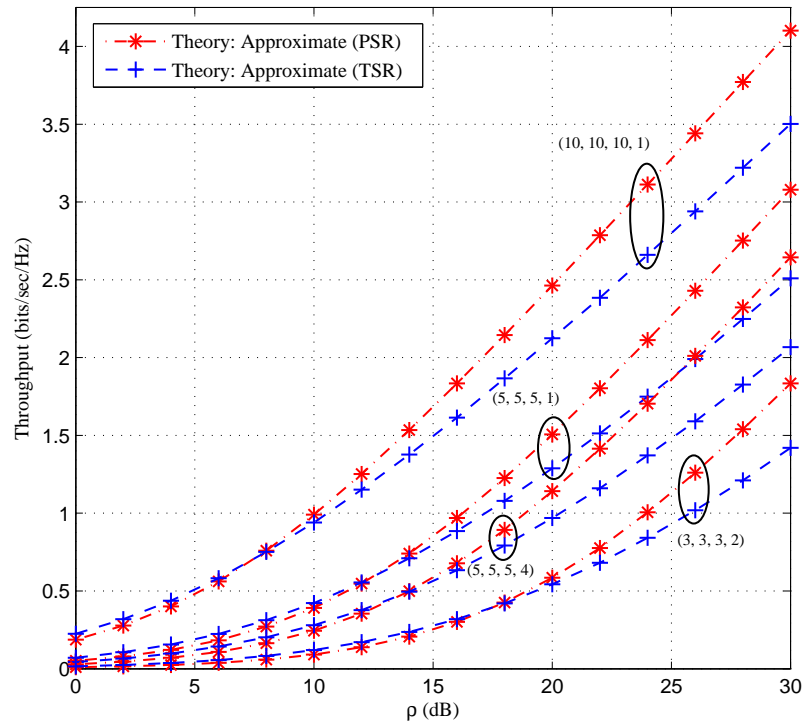

Figure 6: The delay-tolerant (DT) transmission throughput for PSR and TSR with optimal energy harvesting ratio $\beta^{\star}$ and $\alpha^{\star}$ against the first-hop average $\operatorname{SNR} \rho$.

$\min \left(N_{S} N_{R}, N_{R}\left(N_{D}-M\right)\right)$, are $90,20,5$, and 3 , respectively. It is clearly seen in Fig. 6 that the throughput of PSR is superior to that of the TSR, while the difference increases as the SNR increases. Note that the throughput of TSR is slightly higher than that of PSR at low SNR and diversity order. The impact of high diversity order is also investigated in Fig. 6, where higher diversity order clearly provides a substantial

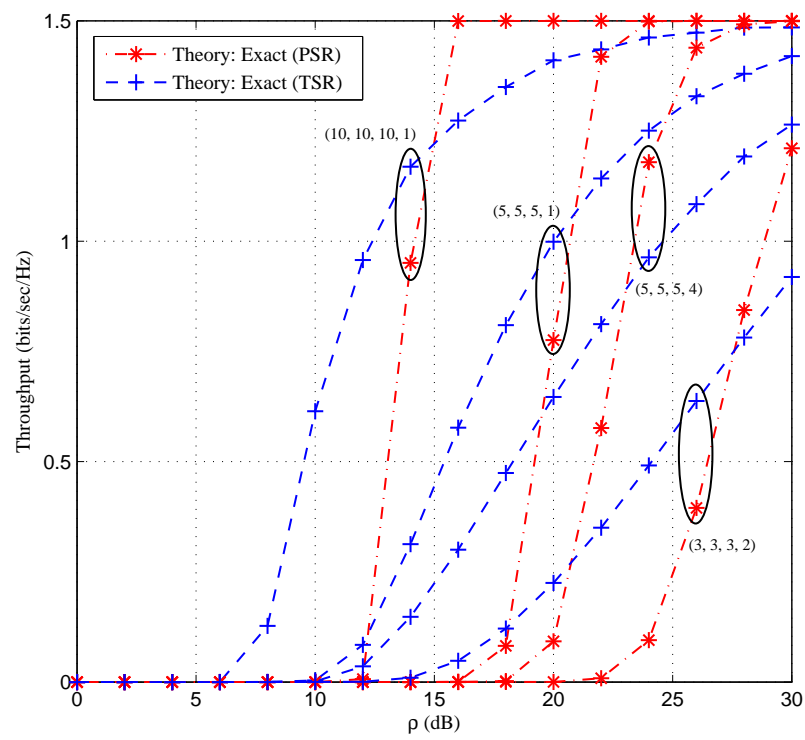

Figure 7: The delay-limited (DL) transmission throughput for PSR and TSR with optimal energy harvesting ratio $\beta^{\star}$ and $\alpha^{\star}$ against the first-hop average SNR $\rho$.

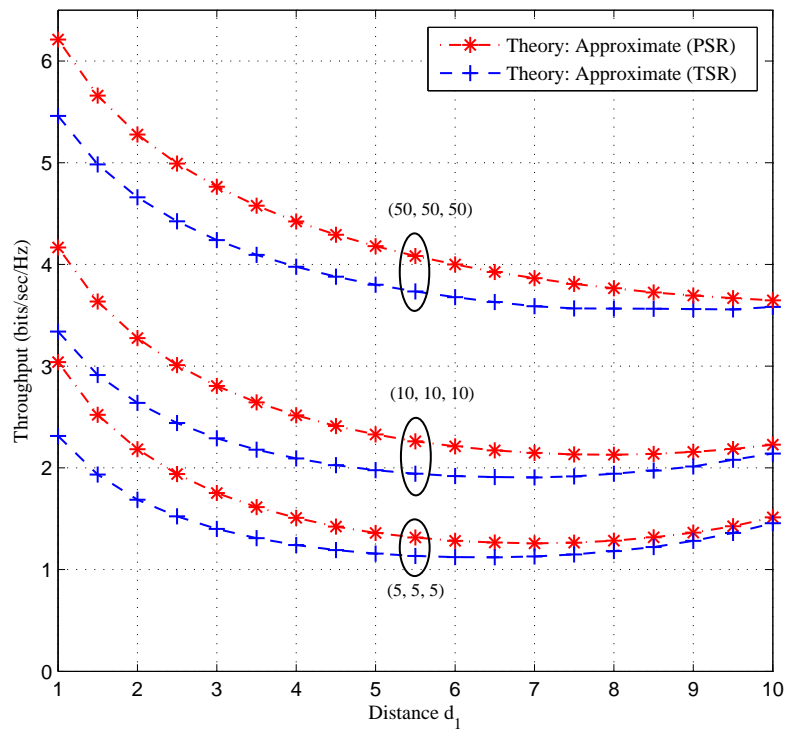

Figure 8: The delay-tolerant (DT) transmission throughput for PSR and TSR with optimal energy harvesting ratio against the distance $d_{1}$, with $d_{2}=11-d_{1}, M=1$, and $\rho=20 \mathrm{~dB}$.

improvement into the system throughput. As ZF is used at the destination, it is to be emphasized that the performance of the setting $(3,3,3,2)$ is equivalent to that of $(3,3,1,0)$ as two antennas (degrees of freedom) at the destination are used for null space projection to eliminate the CCI, i.e., configurations with equivalent $\left(N_{S}, N_{R}, N_{D}-M\right)$ settings will have the same performance.

Fig. 7 compares the throughput performance of the DL 
transmission mode for the TSR and PSR, where the closedform outage probability lower-bound expression (23) is used to evaluate the DL transmission throughput (25) and (26) for the TSR and PSR, respectively. It is observed that the DL throughput of PSR outperforms that of TSR at high SNR. However, at low SNR, TSR outperforms the PSR.

Fig. 8 illustrates the impact of distance on the system performance, namely, the effect of relay location between the source and destination, where the distance between the source and destination is set to $d_{1}+d_{2}=11$. Contrary to the conventional self-powered relaying systems where the relay is placed in the middle between the source and destination, the optimal relay location in wirelessly powered relaying systems is close to the source due to the fact that the relay harvested energy is highly deteriorated by path loss and fading, i.e., higher degradation in the overall throughput is seen as $d_{1}$ increases due to the negative impact of distance between the source and relay on the harvested energy. As information and energy beamforming is performed at the source and relay, it is shown in Fig. 8 that increasing the number of antennas help combat the severe effects of path loss and fading. Therefore, increasing the number of antennas and/or the source power enable longer-distance wireless power transfer.

\section{CONCLUSIONS}

In this paper, the throughput of RF based energy harvesting relaying systems with multiple antenna terminals is investigated for both the delay-limited and delay-tolerant transmission modes, where hop-by-hop information and energy beamforming is considered to maximize the overall SINR. The time-switching receiver and power-splitting receiver are included, and their performances are compared. Based on the proposed upper-bound formula for the overall SINR, closedform analytical expressions for the outage probability and ergodic capacity are derived. In addition, asymptotic expressions were also derived to explicitly reveal some system insights such as the achievable diversity order and array gain. Our results show that there is a significant increase in the optimal throughput due to the increase in diversity order and/or array gain. Furthermore, It is observed that the DT throughput of PSR outperforms that of TSR. However, the DL throughput of PSR outperforms that of TSR at high SNR, while at low SNR, TSR outperforms PSR.

\section{APPENDIX A}

\section{PROOF OF EQUATION (10)}

In order to find an explicit expression for the optimal solution to the optimization problem in (8), the required solution according to the MRC principle is expressed in the following form $\boldsymbol{v}_{R}=\frac{\boldsymbol{P H}_{2} \boldsymbol{v}_{T}}{\left\|\boldsymbol{P} \boldsymbol{H}_{2} \boldsymbol{v}_{T}\right\|}$, where both constraints are included in the desired form, namely, 1) the projection matrix $\boldsymbol{P}$ assures that $\boldsymbol{v}_{R} \perp \boldsymbol{G}$ (owing to the fact that $\boldsymbol{G}^{\dagger} \boldsymbol{v}_{R}=0$ ), where, $\boldsymbol{P}=\boldsymbol{I}_{N_{D}}-\boldsymbol{G}\left(\boldsymbol{G}^{\dagger} \boldsymbol{G}\right)^{-1} \boldsymbol{G}^{\dagger}$, 2) the division by $\left\|\boldsymbol{P} \boldsymbol{H}_{2} \boldsymbol{v}_{T}\right\|$ assures that the norm of $\boldsymbol{v}_{R}$ equals one, i.e., $\left\|\boldsymbol{v}_{R}\right\|=1$. Therefore, (8) can be re-written as

$$
\begin{array}{cc}
\boldsymbol{v}_{T}^{\star}=\underset{\boldsymbol{v}_{T}}{\arg \max } & \left\|\boldsymbol{P} \boldsymbol{H}_{2} \boldsymbol{v}_{T}\right\|^{2} \\
\text { s.t. } & \left\|\boldsymbol{v}_{T}\right\|=1
\end{array} .
$$

Therefore, the problem reduces to the well known squared spectral norm problem (9), that concludes the proof.

\section{APPENDIX B PROOF OF THEOREM 1}

From (20)-(22), it is easy to show that the CDF of the overall SINR upper-bound simplifies to

$$
\begin{gathered}
F_{\gamma^{\text {up }}}\left(\gamma_{T}\right)=\int_{0}^{\infty} F_{\left\|\boldsymbol{H}_{1}\right\|_{2}^{2}}\left(\frac{\gamma_{T}\left(\overline{\gamma_{2}} w+\overline{\gamma_{1}}\right)}{\overline{\gamma_{1} \gamma_{2}} w}\right) f_{\left\|\boldsymbol{P H}_{2}\right\|_{2}^{2}}(w) d w \\
=1-\sum_{i=1}^{\min \left(N_{S}, N_{R}\right)\left(N_{S}+N_{R}\right) i-2 i^{2}} \sum_{j=\left|N_{S}-N_{R}\right|}^{j} \sum_{k=0}^{j} \frac{i^{k} d_{1}(i, j)}{k !}\left(\frac{\gamma_{T}}{\overline{\gamma_{1} \gamma_{2}}}\right)^{k} \\
\times \sum_{m=\left|N_{R}-N_{D}+M\right|}^{\min \left(N_{R}, N_{D}-M\right)} \frac{l_{R}^{m+1} d_{2}(l, m)}{m !} \\
\quad \times \int_{0}^{\infty}\left(\overline{\gamma_{2}} w+\overline{\gamma_{1}}\right)^{k} e^{-i \frac{\gamma_{T}\left(\overline{\gamma_{2}} w+\overline{\gamma_{1}}\right)}{\bar{\gamma}_{1} \gamma_{2}} w} w^{m-k} e^{-l w} d w .
\end{gathered}
$$

Now, once applying the binomial expansion $\left(\overline{\gamma_{2}} w+\overline{\gamma_{1}}\right)^{k}=$ $\sum_{n=0}^{k}\left(\begin{array}{l}k \\ n\end{array}\right)\left(\overline{\gamma_{2}} w\right)^{k-n}{\overline{\gamma_{1}}}^{n}$, equation (46) reduces to

$$
\begin{gathered}
F_{\gamma^{\text {up }}}\left(\gamma_{T}\right)=1-\sum_{i=1}^{\min \left(N_{S}, N_{R}\right)} \sum_{j=\left|N_{S}-N_{R}\right|}^{\left(N_{S}+N_{R}\right) i-2 i^{2}} \sum_{k=0}^{j} \frac{i^{k} d_{1}(i, j)}{k !} \\
\times\left(\frac{\gamma_{T}}{\overline{\gamma_{1}}}\right)^{k} e^{-\frac{i \gamma_{T}}{\gamma_{1}}} \sum_{l=1}^{\min \left(N_{R}, N_{D}-M\right)} \sum_{\left(N_{R}+N_{D}-M\right) l-2 l^{2}}^{l^{m+1} d_{2}(l, m)} \\
\times \sum_{n=0}^{k}\left(\begin{array}{c}
k \\
n
\end{array}\right)\left(\frac{\overline{\gamma_{1}}}{\overline{\gamma_{2}}}\right)^{n !} \underbrace{\int_{0=\left|N_{R}-N_{D}+M\right|}^{\infty} w^{m-n} e^{-\frac{i \gamma_{T}}{\gamma_{2}}-l w} d w}_{I_{1}} .
\end{gathered}
$$

The integral $I_{1}$ is evaluated by utilizing [28, Eq. (3.471.9)], resulting in (23), that concludes the proof.

\section{APPENDIX C PROOF OF EQUATION (28)}

From (27), we have

$$
\begin{gathered}
F_{\gamma^{\text {up } 2}}\left(\gamma_{T}\right)=\operatorname{Pr}\left(\min \left(\gamma_{1}, \gamma_{2}\right)<\gamma_{T}\right) \\
=\operatorname{Pr}\left(\min \left(\overline{\gamma_{1}}\left\|\boldsymbol{H}_{1}\right\|_{2}^{2}, \overline{\gamma_{2}}\left\|\boldsymbol{H}_{1}\right\|_{2}^{2}\left\|\boldsymbol{P} \boldsymbol{H}_{2}\right\|_{2}^{2}\right)<\gamma_{T}\right) \\
=\int_{0}^{\infty} \operatorname{Pr}\left(\min \left(\overline{\gamma_{1}}, \overline{\gamma_{2}}\left\|\boldsymbol{P} \boldsymbol{H}_{2}\right\|_{2}^{2}\right)<\frac{\gamma_{T}}{w}\right) f_{\left\|\boldsymbol{H}_{1}\right\|_{2}^{2}}(w) d w \\
=\int_{0}^{\infty}\left(1-\operatorname{Pr}\left(\overline{\gamma_{1}}>\frac{\gamma_{T}}{w}\right) \operatorname{Pr}\left(\left\|\boldsymbol{P} \boldsymbol{H}_{2}\right\|_{2}^{2}>\frac{\gamma_{T}}{w \overline{\gamma_{2}}}\right)\right) \\
\quad \times f_{\left\|\boldsymbol{H}_{1}\right\|_{2}^{2}}(w) d w .
\end{gathered}
$$

Hence, upon using the asymptotic results of the CDF of the $\boldsymbol{R} \rightarrow \boldsymbol{D}$ link [30, Eq. (27)], equation (48) reduces to 


$$
\begin{aligned}
& F_{\gamma}^{\infty}\left(\gamma_{T}\right)=1-\int_{0}^{\infty} \operatorname{Pr}\left(w>\frac{\gamma_{T}}{\overline{\gamma_{1}}}\right) \\
& \times {\left[1-\frac{\prod_{l=0}^{s_{2}-1} l !}{\prod_{l=0}^{s_{2}-1}\left(t_{2}+l\right) !}\left(\frac{\gamma_{T}}{w \overline{\gamma_{2}}}\right)^{N_{R}\left(N_{D}-M\right)}\right] f_{\left\|\boldsymbol{H}_{1}\right\|_{2}^{2}}(w) d w } \\
&= F_{\left\|\boldsymbol{H}_{1}\right\|_{2}^{2}}\left(\frac{\gamma_{T}}{\overline{\gamma_{1}}}\right)+\frac{\prod_{l=0}^{s_{2}-1} l !}{\prod_{l=0}^{s_{2}-1}\left(t_{2}+l\right) !}\left(\frac{\gamma_{T}}{\overline{\gamma_{2}}}\right)^{N_{R}\left(N_{D}-M\right)} \\
& \times \sum_{i=1}^{s_{1}} \sum_{j=\left|N_{S}-N_{R}\right|}^{\left(N_{S}+N_{R}\right) i-2 i^{2}} \frac{i^{j+1} d_{1}(i, j)}{j !} \int_{I_{2}}^{\int_{\gamma_{T}}^{\infty} w^{j-N_{R}\left(N_{D}-M\right)} e^{-i w} d w} .
\end{aligned}
$$

The integral $I_{2}$ is solved by utilizing [28, Eq. (8.350.2)], resulting in (28), that concludes the proof.

\section{APPENDIX D PROOF OF THEOREM 3}

From (18), moments of the overall SINR upper-bound can be defined as

$$
\begin{aligned}
& \mathbb{E}\left[\left(\gamma^{\text {up }}\right)^{k}\right]=\mathbb{E}\left[\left(\frac{\overline{\gamma_{1} \gamma_{2}}\left\|\boldsymbol{H}_{1}\right\|_{2}^{2}\left\|\boldsymbol{P} \boldsymbol{H}_{2}\right\|_{2}^{2}}{\overline{\gamma_{2}}\left\|\boldsymbol{P} \boldsymbol{H}_{2}\right\|_{2}^{2}+\overline{\gamma_{1}}}\right)^{k}\right] \\
& =\mathbb{E}\left[\left(\overline{\gamma_{1}}\left\|\boldsymbol{H}_{1}\right\|_{2}^{2}\right)^{k}\right] \mathbb{E}\left[\left(\frac{\overline{\gamma_{2}}\left\|\boldsymbol{P} \boldsymbol{H}_{2}\right\|_{2}^{2}}{\overline{\gamma_{2}}\left\|\boldsymbol{P} \boldsymbol{H}_{2}\right\|_{2}^{2}+\overline{\gamma_{1}}}\right)^{k}\right] .
\end{aligned}
$$

Where due to independence between the random variables $\left\|\boldsymbol{H}_{1}\right\|_{2}^{2}$ and $\left\|\boldsymbol{P} \boldsymbol{H}_{2}\right\|_{2}^{2}$, the moments of the overall SINR upperbound can be easily simplified to (50). To this end, by utilizing the PDFs of $\left\|\boldsymbol{H}_{1}\right\|_{2}^{2}$ and $\left\|\boldsymbol{P} \boldsymbol{H}_{2}\right\|_{2}^{2}$ in (21)-(22), and with the help of [29, Eq. (6.1.1)] and [28, Eq. (9.211.4)], respectively, we have

$$
\begin{gathered}
\mathbb{E}\left[\left(\overline{\gamma_{1}}\left\|\boldsymbol{H}_{1}\right\|_{2}^{2}\right)^{k}\right]=\bar{\gamma}^{k} \sum_{i=1}^{\min \left(N_{S}, N_{R}\right)} \sum_{j=\left|N_{S}-N_{R}\right|}^{\left(N_{S}+N_{R}\right) i-2 i^{2}} \\
\times \frac{i^{j+1} d_{1}(i, j)}{j !} \int_{0}^{\infty} x^{k+j} e^{-i x} d x \\
=\bar{\gamma}_{1}^{k} \sum_{i=1}^{\min \left(N_{S}, N_{R}\right)} \sum_{j=\left|N_{S}-N_{R}\right|}^{\left(N_{S}+N_{R}\right) i-2 i^{2}} \frac{d_{1}(i, j)}{j !}\left(\frac{1}{i}\right)^{k} \Gamma(k+j+1)
\end{gathered}
$$

and

$$
\begin{gathered}
\mathbb{E}\left[\left(\frac{\overline{\gamma_{2}}\left\|\boldsymbol{P H}_{2}\right\|_{2}^{2}}{\overline{\gamma_{2}}\left\|\boldsymbol{P} \boldsymbol{H}_{2}\right\|_{2}^{2}+\overline{\gamma_{1}}}\right)^{k}\right] \\
=\sum_{l=1}^{\min \left(N_{R}, N_{D}-M\right)} \sum_{\left(N_{R}+N_{D}-M\right) l-2 l^{2}}^{m=\left|N_{R}-N_{D}+M\right|} \\
\times \frac{l^{m+1} d_{2}(l, m)}{m !} \int_{0}^{\infty}\left(\frac{\overline{\gamma_{2}} y}{\overline{\gamma_{1}}+\overline{\gamma_{2}} y}\right)^{k} y^{m} e^{-l y} \\
=\sum_{l=1}^{\min \left(N_{R}, N_{D}-M\right)} \sum_{\left(N_{R}+N_{D}-M\right) l-2 l^{2}} \frac{d_{2}(l, m)}{m !} \\
\times \Gamma(k+m+1) \Psi\left(k,-m ; \frac{l \overline{\gamma_{1}}}{\overline{\gamma_{2}}}\right) .
\end{gathered}
$$

Hence, upon substituting (51) and (52) into (50), we arrive at (33), that concludes the proof.

\section{APPENDIX E}

\section{PROOF OF THEOREM 5}

By utilizing the PDFs of $\left\|\boldsymbol{H}_{1}\right\|_{2}^{2}$ and $\left\|\boldsymbol{P} \boldsymbol{H}_{2}\right\|_{2}^{2}$ in (21)-(22), the expectations in (37) and (38) can be (respectively) derived as

$$
\begin{aligned}
\mathbb{E}\left[\ln \left(\left\|\boldsymbol{H}_{1}\right\|_{2}^{2}\right)\right]= & \sum_{i=1}^{\min \left(N_{S}, N_{R}\right)} \sum_{j=\left|N_{S}-N_{R}\right|}^{\left(N_{S}+N_{R}\right) i-2 i^{2}} \\
& \times \frac{i^{j+1} d_{1}(i, j)}{j !} \int_{0}^{\infty} x^{j} \ln x e^{-i x} d x
\end{aligned}
$$

and

$$
\begin{aligned}
& \mathbb{E}\left[\ln \left(\overline{\gamma_{2}}\left\|\boldsymbol{P} \boldsymbol{H}_{2}\right\|_{2}^{2}\right)\right]=\ln \left(\overline{\gamma_{2}}\right)+\sum_{l=1}^{\min \left(N_{R}, N_{D}-M\right)} \\
& \times \sum_{m=\left|N_{R}-N_{D}+M\right|}^{\left(N_{R}+N_{D}-M\right) l-2 l^{2}} \frac{l^{m+1} d_{2}(l, m)}{m !} \int_{0}^{\infty} y^{m} \ln y e^{-l y} .
\end{aligned}
$$

The integrals in (53) and (54) are solved by utilizing [28, Eq. (4.352.1)], resulting in (37) and (38), while (39) is solved with the help of [31, Lemma 1] which says, for any $x \geq 0$, we have

$$
\mathbb{E}[\ln (1+x)]=\int_{0}^{\infty} \frac{1}{z}\left(1-\mathcal{M}_{x}(z)\right) e^{-z} d z
$$

where $\mathcal{M}_{x}(z)=\mathbb{E}\left[e^{-z x}\right]$ is the moment generating function (MGF) of the random variable $x=\frac{\overline{\gamma_{2}}}{\gamma_{1}}\left\|\boldsymbol{P} \boldsymbol{H}_{2}\right\|_{2}^{2}$, which is given as [22, Eq. (20)]

$$
\mathcal{M}_{x}(z)=\sum_{l=1}^{\min \left(N_{R}, N_{D}-M\right)} \sum_{m=\left|N_{R}-N_{D}+M\right|}^{\left(N_{R}+N_{D}-M\right) l-2 l^{2}} \frac{d_{2}(l, m)}{\left(\frac{\overline{\gamma_{2}}}{l \overline{\gamma_{1}}} z+1\right)^{m+1}} \text {. }
$$


In order to attain a closed-form solution for (55), an alternative form for the MGF of $x$ may be found similarly to [32, Eq. (26)], as follows

$$
\begin{aligned}
& \mathcal{M}_{x}(z)=1-\frac{\overline{\gamma_{2}}}{\overline{\gamma_{1}}} \sum_{l=1}^{\min \left(N_{R}, N_{D}-M\right)} \sum_{m=\left|N_{R}-N_{D}+M\right|}^{\left(N_{R}+N_{D}-M\right) l-2 l^{2}} \\
& \times \sum_{n=0}^{m} \frac{d_{2}(l, m)}{l} \frac{z}{\left(\frac{\overline{\gamma_{2}}}{l \overline{\gamma_{1}}} z+1\right)^{n+1}} .
\end{aligned}
$$

Therefore, upon substituting the MGF (57) into (55), we arrive at

$$
\begin{gathered}
\mathbb{E}\left[\ln \left(1+\frac{\overline{\gamma_{2}}}{\overline{\gamma_{1}}}\left\|\boldsymbol{P} \boldsymbol{H}_{2}\right\|_{2}^{2}\right)\right]=\frac{\overline{\gamma_{2}}}{\overline{\gamma_{1}}} \sum_{l=1}^{\min \left(N_{R}, N_{D}-M\right)} \\
\times \sum_{m=\left|N_{R}-N_{D}+M\right|}^{\left(N_{R}+N_{D}-M\right) l-2 l^{2}} \sum_{n=0}^{m} \frac{1}{l} d_{2}(l, m) \underbrace{\int_{0}^{\infty} \frac{1}{\left(\frac{\overline{\gamma_{2}}}{l \overline{\gamma_{1}}} z+1\right)^{n+1}} e^{-z} d z}_{I_{3}} \\
=\sum_{m=\left|N_{R}-N_{D}+M\right|}^{\min \left(N_{R}, N_{D}-M\right)} \sum_{\left(N_{R}+N_{D}-M\right) l-2 l^{2}}^{m} d_{2}(l, m) \\
\times \Psi\left(1,1-n ; \frac{l \overline{\gamma_{1}}}{\overline{\gamma_{2}}}\right) .
\end{gathered}
$$

The integral $I_{3}$ is solved by utilizing [28, Eq. (9.211.4)], which upon using the fact that $\Psi\left(1,1-n ; \frac{l \overline{\gamma_{1}}}{\overline{\gamma_{2}}}\right)=$ $e^{\frac{l \overline{\gamma_{1}}}{\bar{\gamma}_{2}}} \mathrm{E}_{1+n}\left(\frac{l \overline{\gamma_{1}}}{\overline{\gamma_{2}}}\right)$, reduces to (39), that concludes the proof.

\section{REFERENCES}

[1] X. Chen, Z. Zhang, H.-H. Chen, and H. Zhang, "Enhancing wireless information and power transfer by exploiting multi-antenna techniques," IEEE Commun. Mag., vol. 53, no. 4, pp. 133-141, April 2015.

[2] Z. Ding, C. Zhong, D. Ng, M. Peng, H. Suraweera, R. Schober, and H. Poor, "Application of smart antenna technologies in simultaneous wireless information and power transfer," IEEE Commun. Mag., vol. 53, no. 4, pp. 86-93, April 2015.

[3] Y. Gu and S. Aissa, "RF-based energy harvesting in decode-and-forward relaying systems: Ergodic and outage capacities," IEEE Trans. Wireless Commun., vol. 14, no. 11, pp. 6425-6434, Nov. 2015.

[4] A. Nasir, X. Zhou, S. Durrani, and R. Kennedy, "Relaying protocols for wireless energy harvesting and information processing," IEEE Trans. Wireless Commun., vol. 12, no. 7, pp. 3622-3636, July 2013.

[5] M. Ku, W. Li, Y. Chen, and K. Liu, "Advances in energy harvesting communications: Past, present, and future challenges," IEEE Commun. Surveys Tuts., vol. 18, no. 2, pp. 1384-1412, April 2016.

[6] X. Lu, P. Wang, D. Niyato, D. Kim, and Z. Han, "Wireless charging technologies: Fundamentals, standards, and network applications," IEEE Commun. Surveys Tuts., vol. 18, no. 2, pp. 1413-1452, April 2016.

[7] H. Chen, Y. Li, J. L. Rebelatto, B. F. Uchoa-Filho, and B. Vucetic, "Harvest-then-cooperate: Wireless-powered cooperative communications," IEEE Transactions on Signal Processing, vol. 63, no. 7, pp. 1700-1711, April 2015.

[8] A. A. Nasir, X. Zhou, S. Durrani, and R. A. Kennedy, "Wireless-powered relays in cooperative communications: Time-switching relaying protocols and throughput analysis," IEEE Transactions on Communications, vol. 63, no. 5, pp. 1607-1622, May 2015.

[9] Y. Chen, "Energy harvesting AF relaying in the presence of interference and nakagami-m fading," IEEE Trans. Wireless Commun., vol. 15, no. 2, pp. 1008-1017, Feb. 2016.
[10] Z. Ding, S. Perlaza, I. Esnaola, and H. Poor, "Power allocation strategies in energy harvesting wireless cooperative networks," IEEE Trans. Wireless Commun., vol. 13, no. 2, pp. 846-860, Feb. 2014.

[11] D. Michalopoulos, H. Suraweera, and R. Schober, "Relay selection for simultaneous information transmission and wireless energy transfer: A tradeoff perspective," IEEE J. Sel. Areas Commun., vol. 33, no. 8, pp. 1578-1594, Aug. 2015

[12] I. Krikidis, "Relay selection in wireless powered cooperative networks with energy storage," IEEE J. Sel. Areas Commun., vol. 33, no. 12, pp. 2596-2610, Dec. 2015.

[13] C. Zhong, H. Suraweera, G. Zheng, I. Krikidis, and Z. Zhang, "Wireless information and power transfer with full duplex relaying," IEEE Trans Commun., vol. 62, no. 10, pp. 3447-3461, Oct. 2014.

[14] R. Zhang and C. K. Ho, "MIMO broadcasting for simultaneous wireless information and power transfer," IEEE Trans. Wireless Commun., vol. 12, no. 5, pp. 1989-2001, May 2013.

[15] W. Huang, H. Chen, Y. Li, and B. Vucetic, "On the performance of multiantenna wireless-powered communications with energy beamforming," IEEE Trans. Veh. Technol., vol. 65, no. 3, pp. 1801-1808, March 2016.

[16] L. Liu, R. Zhang, and K.-C. Chua, "Multi-antenna wireless powered communication with energy beamforming," IEEE Trans. Commun., vol. 62, no. 12, pp. 4349-4361, Dec. 2014.

[17] G. Zhu, C. Zhong, H. Suraweera, G. Karagiannidis, Z. Zhang, and T. Tsiftsis, "Wireless information and power transfer in relay systems with multiple antennas and interference," IEEE Trans. Commun., vol. 63 , no. 4, pp. 1400-1418, April 2015.

[18] Y. Zeng and R. Zhang, "Full-duplex wireless-powered relay with selfenergy recycling," IEEE Wireless Commun. Lett., vol. 4, no. 2, pp. 201204, April 2015.

[19] P. Dighe, R. Mallik, and S. Jamuar, "Analysis of transmit-receive diversity in rayleigh fading," IEEE Trans. Commun., vol. 51, no. 4, pp. 694-703, April 2003.

[20] A. Maaref and S. Aissa, "Closed-form expressions for the outage and ergodic shannon capacity of MIMO MRC systems," IEEE Trans. Commun., vol. 53, no. 7, pp. 1092-1095, July 2005.

[21] H. Suraweera, I. Krikidis, G. Zheng, C. Yuen, and P. Smith, "Lowcomplexity end-to-end performance optimization in MIMO full-duplex relay systems," IEEE Trans. Wireless Commun., vol. 13, no. 2, pp. 913927, Feb. 2014

[22] G. Amarasuriya, C. Tellambura, and M. Ardakani, "Performance analysis of hop-by-hop beamforming for dual-hop MIMO AF relay networks," IEEE Trans. Commun., vol. 60, no. 7, pp. 1823-1837, July 2012.

[23] M. Hasna and M.-S. Alouini, "End-to-end performance of transmission systems with relays over Rayleigh-fading channels," IEEE Trans. Wireless Commun., vol. 2, no. 6, pp. 1126-1131, Nov. 2003.

[24] S. Ikki and S. Aissa, "Performance analysis of two-way amplify-andforward relaying in the presence of co-channel interferences," IEEE Trans. Commun., vol. 60, no. 4, pp. 933-939, April 2012.

[25] A. Almradi and K. Hamdi, "The performance of wireless powered MIMO relaying systems with energy beamforming," in Proc. IEEE ICC'16, May 2016, pp. 1-6.

[26] D. da Costa and S. Aissa, "Capacity analysis of cooperative systems with relay selection in nakagami-m fading," IEEE Commun. Lett., vol. 13, no. 9, pp. 637-639, Sept. 2009.

[27] M. Xia, C. Xing, Y.-C. Wu, and S. Aissa, "Exact performance analysis of dual-hop semi-blind AF relaying over arbitrary nakagami-m fading channels," IEEE Trans. Wireless Commun., vol. 10, no. 10, pp. 34493459, Oct. 2011.

[28] I. S. Gradshteyn and I. M. Ryzhik, Tables of Integrals, Series and Products. 6th ed. San Diego, CA, USA: Academic, 2000.

[29] M. Abramowitz and I. A. Stegun, Handbook of Mathematical Functions. New York: Dover, 1965.

[30] S. Jin, M. McKay, X. Gao, and I. Collings, "Asymptotic SER and outage probability of MIMO MRC in correlated fading," IEEE Signal Process. Lett., vol. 14, no. 1, pp. 9-12, Jan. 2007.

[31] K. Hamdi, "Capacity of MRC on correlated rician fading channels," IEEE Trans. Commun., vol. 56, no. 5, pp. 708-711, May 2008.

[32] A. Almradi and K. Hamdi, "Ergodic capacity analysis of MIMO full duplex relaying with imperfect CSI," in Proc. IEEE GLOBECOM'15, Dec. 2015, pp. 1-6. 